\title{
Sur la cohomologie galoisienne des corps $p$-adiques
}

Bulletin de la S. M. F., tome 126, no 4 (1998), p. 563-600

<http://www.numdam.org/item?id=BSMF_1998_126_4_563_0>

(C) Bulletin de la S. M. F., 1998, tous droits réservés.

L'accès aux archives de la revue «Bulletin de la S. M. F. » (http: //smf.emath.fr/Publications/Bulletin/Presentation.html) implique l'accord avec les conditions générales d'utilisation (http://www.numdam.org/ conditions). Toute utilisation commerciale ou impression systématique est constitutive d'une infraction pénale. Toute copie ou impression de ce fichier doit contenir la présente mention de copyright.

\section{Numdam}


Bull. Soc. math. France,

126,1998 , p. 563-600.

\title{
SUR LA COHOMOLOGIE GALOISIENNE DES CORPS $p$-ADIQUES
}

\author{
PAR LAURENT HERR $(*)$
}

RÉSUMÉ. - Ce travail fait suite à l'article de J.-M. Fontaine paru dans la Grothendieck Festschrift, où il construit une équivalence entre la catégorie des représentations $p$-adiques du groupe de Galois absolu $G_{K}$ d'un corps local $K$ d'inégale caractéristique $(0, p>0)$ et une catégorie de modules sur un certain anneau, munis de deux opérateurs aux propriétés particulières. Nous donnons ici à l'aide de ces nouveaux objets une construction explicite des groupes de cohomologie galoisienne d'une représentation $\mathbb{Z}_{p}$-adique de $p$-torsion de $G_{K}$. Lorsque $K$ est une extension finie de $\mathbb{Q}_{p}$, nous montrons ensuite comment on peut retrouver à partir de là les résultats classiques de Tate concernant ces groupes : la finitude et le calcul de la caractéristique d'Euler-Poincaré. Les méthodes utilisées semblent être plus simples que les arguments cohomologiques habituels, ne serait-ce que parce que l'on ne se sert pas de la théorie sophistiquée du corps de classe local et que tout est essentiellement explicite. Nous obtenons aussi au passage des résultats importants concernant la structure des modules associés aux représentations, ainsi qu'une filtration en trois crans sur leur cohomologie.

Abstract. - On Galois COHOMOlogy OF $p$-AdiC Fields. - This work follows J.-M. Fontaine's paper in the Grothendieck Festschrift, where he constructs an equivalence between the category of $\mathbb{Z}_{p}$-adic representations of the absolute Galois group $G_{K}$ of a local field $K$ of mixed characteristic $(0, p>0)$ and a category of modules over a certain ring, endowed with two operators satisfying special properties. We give here an explicit construction of the cohomology groups of a $\mathbb{Z}_{p}$-adic representation of $G_{K}$ killed by a power of $p$, using these new objects. When $K$ is a finite extension of $\mathbb{Q}_{p}$, we show then how one can find again Tate's classical results about these groups : the finiteness and the calculation of the Euler-Poincaré characteristic. The methods used seem to be rather simpler than the standard cohomological arguments because we don't need sophisticated theories like local class field theory and everything is essentially explicit. One gets also interesting information about the structure of the modules associated with representations and a 3-step filtration on their Galois cohomology.

(*) Texte reçu le 9 janvier 1998, révisé le 23 septembre 1998, accepté le 9 octobre 1998. L. HerR, Laboratoire de Mathématiques Pures, Université Bordeaux 1, 351 cours de la Libération, 33405, Talence CEDEX (France).

E-mail : herr@math.u-bordeaux.fr.

Mots clés : cohomologie galoisienne, corps $p$-adiques.

Classification AMS : 11S 25, 11 S 15, 11S 31, 14F 30.

BULlETIN DE LA SOCIÉTÉ MATHÉMATIQUE DE FRANCE 0037-9484/1998/563/\$5.00

(C) Société mathématique de France 


\section{Introduction}

Dans tout ce texte, $K$ est un corps complet pour une valuation discrète, de caractéristique 0 , à corps résiduel $k$ parfait de caractéristique $p>0$. On choisit une clôture séparable $\bar{K}$ de $K$, on note son corps résiduel $\bar{k}$ et on pose

$$
G_{K}=\operatorname{Gal}(\bar{K} / K) .
$$

On appelle représentation $\mathbb{Z}_{p}$-adique de $G_{K}$ la donnée d'un $\mathbb{Z}_{p}$-module $V$ de type fini muni d'une action linéaire continue de $G_{K}$. Ces représentations forment une catégorie abélienne que l'on note

$$
\operatorname{Rep}_{\mathbb{Z}_{p}}\left(G_{K}\right) \text {. }
$$

Si $V$ est annulé par une puissance de $p$, on dit que la représentation est de $p$-torsion. Si $V$ est un $\mathbb{F}_{p}$-espace vectoriel, on parle de représentation $\bmod p$ de $G_{K}$. On note

$$
\operatorname{Rep}_{p \text {-tor }}\left(G_{K}\right) \quad\left(\operatorname{resp} . \boldsymbol{R e p}_{\mathbb{F}_{p}}\left(G_{K}\right)\right)
$$

la sous-catégorie pleine de $\boldsymbol{R e p}_{\mathbb{Z}_{p}}\left(G_{K}\right)$ formée des représentations de $p$-torsion $(\operatorname{resp} . \bmod p)$.

Soit $V$ une représentation de $p$-torsion de $G_{K}$. On peut définir ses groupes de cohomologie $H^{i}\left(G_{K}, V\right)$. Rappelons les résultats classiques suivants :

ThÉORÈme A (annulation de la cohomologie, [Se2, chap. II, prop. 12]). Les groupes $H^{i}\left(G_{K}, V\right)$ sont nuls pour $i \geq 3$.

ThÉorème B (finitude de la cohomologie, cf. [Tat]). - Lorsque $k$ est fini, les groupes $H^{i}\left(G_{K}, V\right)$ le sont aussi et la caractéristique d'EulerPoincaré vaut :

$$
\chi(V)=\prod_{1 \leq i \leq 2} \operatorname{card}\left(H^{i}\left(G_{K}, V\right)\right)^{(-1)^{i}}=p^{-\left[K: \mathbb{Q}_{p}\right] \operatorname{long}_{\mathbb{Z}_{p}}(V)} .
$$

ThÉorÈme C (dualité locale, $c f$. [Tat]). - Soit $V^{*}(1)=\operatorname{Hom}\left(V, \mu_{p^{\infty}}\right)$ le dual de $V$ tordu à la Tate. Si $k$ est fini, il existe un isomorphisme canonique de $H^{2}\left(G_{K}, \mu_{p^{\infty}}\right)$ sur $\mathbb{Q}_{p} / \mathbb{Z}_{p}$. Pour $i \in\{0,1,2\}$, le cupproduit induit alors une dualité parfaite entre les groupes $H^{i}\left(G_{K}, V\right)$ et $H^{2-i}\left(G_{K}, V^{*}(1)\right)$.

Les démonstrations classiques de ces résultats (voir [Se2] ou [Mil]), qui utilisent de façon déterminante l'étude du groupe de Brauer, sont longues, difficiles et peu «explicites». On se propose ici d'utiliser l'équivalence de catégorie décrite par J.-M. Fontaine [Fo1] dans la Grothendieck Festschrift, 
entre représentions $\mathbb{Z}_{p}$-adiques de $G_{K}$ et $\left\langle\Phi-\Gamma_{K}\right.$-module étales》 pour associer à $V$ un complexe canonique qui calcule sa cohomologie. On étudie en détail quelques propriétés de ce complexe, et on en déduit une démonstration 《élémentaire 》 des théorèmes $\mathrm{A}$ et $\mathrm{B}$. On obtient également une décomposition canonique des groupes de cohomologie $H^{i}\left(G_{K}, V\right)$. Nous montrerons dans une publication ultérieure comment on peut aussi retrouver le théorème $\mathrm{C}$ par ce type de techniques.

Lorsque le corps résiduel de $K$ est fini, on peut généraliser les résultats précédents à la cohomologie continue des représentations $\mathbb{Z}_{p}$-adiques quelconques, ou même à celle des représentations p-adiques de $G_{K}$, i.e. des $\mathbb{Q}_{p}$-espaces vectoriels de dimension finie munis d'une action linéaire continue de $G_{K}$, de la façon suivante.

Rappelons qu'on dit qu'un système projectif $\left(A_{n}, \pi_{n}\right)_{n \in \mathbb{N}}$ vérifie la condition de Mittag-Leffler si, pour tout entier naturel $n$, l'image des applications de transition $A_{n+m} \rightarrow A_{n}$ est constante pour $m$ assez grand.

Si $T$ est une représentation $\mathbb{Z}_{p}$-adique de $G_{K}$ et $i$ un entier naturel, le théorème de finitude $\mathrm{B}$ entraîne alors que le système projectif des $H^{i}\left(G_{K}, T / p^{n} T\right)$ vérifie la condition de Mittag-Leffler et de ce fait l'application naturelle

$$
H^{i}\left(G_{K}, T\right) \longrightarrow \varliminf_{n \geq 1} H^{i}\left(G_{K}, T / p^{n} T\right)
$$

est un isomorphisme. De plus, si $V$ est une représentation $p$-adique de $G_{K}$ et $T$ un réseau de $V$ stable par l'action de $G_{K}$, alors, pour tout entier naturel $i$, on a :

$$
H^{i}\left(G_{K}, V\right) \simeq \mathbb{Q}_{p} \otimes_{\mathbb{Z}_{p}} H^{i}\left(G_{K}, T\right) .
$$

Par passage à la limite et extension des scalaires, on déduit donc des théorèmes $A$ à $C$, le résultat suivant :

THÉORÈme. - On suppose $k$ fini. Soit $T$ une représentation $\mathbb{Z}_{p}$-adique de $G_{K}$. Alors ses groupes de cohomologie continue $H^{i}\left(G_{K}, T\right)$ sont des $\mathbb{Z}_{p^{-}}$ module de type fini, nuls pour $i \geq 3$ et si $T$ est sans torsion, on a la relation:

$$
\sum_{i \in \mathbb{N}}(-1)^{i} \operatorname{rang}_{\mathbb{Z}_{p}} H^{i}\left(G_{K}, T\right)=-\left[K: \mathbb{Q}_{p}\right] \operatorname{rang}_{\mathbb{Z}_{p}} T .
$$

De plus, il existe un isomorphisme canonique de $H^{2}\left(G_{K}, \mathbb{Z}_{p}(1)\right)$ sur $\mathbb{Z}_{p}$ et, si $T^{*}(1)$ désigne le dual de $T$ tensorisé par $\mathbb{Z}_{p}(1)$, alors, pour $i \in\{0,1,2\}$, le cup-produit induit une dualité parfaite entre les $\mathbb{Z}_{p}$-modules $H^{i}\left(G_{K}, T\right)$ et $H^{2-i}\left(G_{K}, T^{*}(1)\right)$.

Si $V$ est une représentation p-adique de $G_{K}$, on a les résultats analogues en remplaçant $\mathbb{Z}_{p}$ par $\mathbb{Q}_{p}$. 
Voici le plan de cet article :

- $\S 1$ : on rappelle la construction de Fontaine qui permet d'associer à toute représentation de $p$-torsion $V$ de $G_{K}$ un $« \Phi-\Gamma_{K}$-module étale de torsion $M \operatorname{sur} \mathcal{O}_{\mathcal{E}(K)}{ }$;

- $\S 2$ : on construit en utilisant $M$ un complexe canonique de longueur 2 qui calcule la cohomologie galoisienne de $V$, ce qui prouve le théorème $\mathrm{A}$;

- $\S 3$ : on construit un inverse à gauche $\psi_{M}$ du Frobenius $\phi_{M}$ agissant sur $M$ et on étudie quelques unes de ses propriétés;

- $\S 4$ : on montre comment retrouver le théorème $\mathrm{B}$ à l'aide des résultats du paragraphe précédent;

- $\S 5$ : on utilise des calculs de ramification pour prouver un résultat énoncé au paragraphe 3 qui est le cœur technique du théorème sur la structure du noyau de $\psi_{M}$.

Cet article est une version remaniée par endroits, d'après des suggestions de J.-M. Fontaine, de la thèse de l'auteur sous sa direction. Plus précisément, la partie 3.3 donne une présentation plus agréable de la structure du noyau de l'opérateur $\psi_{M}$, entre autre par un usage plus poussé de la notion de «réseau »; le $\S 5$ généralise un peu l'étude initiale de l'action du groupe $\Gamma_{K}$ sur le quotient d'un corps de séries formelles de caractéristique $p$ par les puissances $p$-ièmes. Cet article a probablement gagné ainsi en clarté et en concision.

Je tiens donc à terminer cette introduction en remerciant chaleureusement J.-M. Fontaine d'avoir eu la patience de guider mes pas dans ce travail, qui, sans lui, n'aurait sans doute pas vu le jour.

\section{Préliminaires}

\subsection{Construction de quelques anneaux.}

Pour les détails on renvoie le lecteur à [Fo1, A3.1-3.2].

\subsubsection{Le corps $\operatorname{Fr} R$.}

1.1.1.1. Définition. - On note $C$ le complété de $\bar{K}$ pour la topologie $p$ adique, $v_{C}$ la valuation de $C$ normalisée par $v_{C}(p)=1$ et $\operatorname{Fr} R$ l'ensemble des suites $\left(x^{(n)}\right)_{n \in \mathbb{N}}$ de $C$ telles que, pour tout $n \in \mathbb{N}$,

$$
\left(x^{(n+1)}\right)^{p}=x^{(n)} .
$$

On munit Fr $R$ d'une addition et d'une multiplication en posant pour tout $x=\left(x^{(n)}\right)_{n \in \mathbb{N}}$ et tout $y=\left(y^{(n)}\right)_{n \in \mathbb{N}}$ dans $\operatorname{Fr} R$ :

$$
x y=\left(x^{(n)} y^{(n)}\right)_{n \in \mathbb{N}}, \quad x+y=\left(\lim _{m \rightarrow+\infty}\left(x^{(m+n)}+y^{(m+n)}\right)^{p^{m}}\right)_{n \in \mathbb{N}} .
$$

TOME $126-1998-\mathrm{N}^{\circ} 4$ 
On obtient alors un corps algébriquement clos de caractéristique $p$, complet pour la valuation $v$ définie par

$$
v(x)=v_{C}\left(x^{(0)}\right)
$$

et son corps résiduel s'identifie à $\bar{k}$ de la façon suivante : à $x \in \bar{k}$, on associe l'élément $\left([x]^{p^{-n}}\right)_{n \in \mathbb{N}}$ de Fr $R$, où $[x]$ est le relèvement de Teichmüller de $x$ dans $C$. On note $R$ l'anneau de la valuation. Le groupe $G_{K}=\operatorname{Gal}(\bar{K} / K)$ opère continûment sur Fr $R$.

1.1.1.2. Lien avec le corps des normes. - Dans tout cet article, sauf dans le $\S 5, K_{\infty}$ désigne la $\mathbb{Z}_{p}$-extension cyclotomique de $K$ contenue dans $\bar{K}$; son corps résiduel $k_{\infty}$ est une extension finie de $k$. Pour tout $n \in \mathbb{N}$, on note $K^{(n)}$ l'unique extension de $K$ de degré $p^{n}$ contenue dans $K_{\infty}$. Pour une extension de corps quelconque $L / T$, on désigne par $\mathcal{E}_{L / T}$ l'ensemble ordonné filtrant des extensions finies de $T$ contenues dans $L$.

Pour toute extension finie $L$ de $K_{\infty}$, on peut définir le corps des normes $E_{K}(L)$ de $L / K$ (pour toute la théorie, on renvoie à [Win]). Rappelons qu'en tant qu'ensemble,

$$
E_{K}(L)=\lim _{T \in \mathcal{E}_{L / K}} T
$$

les applications de transition étant les normes. Pour $x=\left(x_{T}\right)_{T \in \mathcal{E}_{L / K}}$ et $y=\left(y_{T}\right)_{\mathcal{E}_{L / K}} \in E_{K}(L)$, on définit la somme et le produit par

$$
\begin{gathered}
x y=\left(x_{T} y_{T}\right)_{T \in \mathcal{E}_{L / K}}, \\
x+y=\left(\lim _{T^{\prime} \in \mathcal{E}_{L / T}} N_{T^{\prime} / T}\left(x_{T^{\prime}}+y_{T^{\prime}}\right)\right)_{T \in \mathcal{E}_{L / K}},
\end{gathered}
$$

la limite étant prise suivant le filtre des sections de $\mathcal{E}_{L / T}$. Alors, $E_{K}(L)$ est un corps de caractéristique $p$, complet pour la valuation discrète donnée par

$$
v(x)=v_{C}\left(x_{K}\right)
$$

et son corps résiduel s'identifie à celui de $L$.

Cette construction est en fait fonctorielle et donne, par passage à la limite inductive, une équivalence entre la catégorie des extensions algébriques de $K_{\infty}$ et celle des extensions séparables de $E_{K}\left(K_{\infty}\right)$. Le corps

$$
E_{K}(\bar{K})=\underset{L \in \operatorname{\mathcal {E}}_{\bar{K} / K_{\infty}}}{\lim } E_{K}(L)
$$


est donc une clôture séparable de $E_{K}\left(K_{\infty}\right)$. Le groupe $G_{K}$ opère de façon continue sur $E_{K}(\bar{K})$ par fonctorialité; cette action permet d'identifier $\operatorname{Gal}\left(E^{\text {sep }} / E_{K}\left(K_{\infty}\right)\right)$ à $\operatorname{Gal}\left(\bar{K} / K_{\infty}\right)$ et, pour tout $L \in \mathcal{E}_{\bar{K} / K_{\infty}}$, on a :

$$
E_{K}(L)=\left(E_{K}(\bar{K})\right)^{\operatorname{Gal}(\bar{K} / L)} .
$$

Il existe de plus un plongement continu et $G_{K}$-équivariant $j_{K}$ de $E_{K}(\bar{K})$ dans $\operatorname{Fr} R$ qui est défini de la façon suivante : soient $L$ une extension finie de $K_{\infty}$ contenue dans $\bar{K}$ et $L^{\mathrm{mr}}$ l'extension maximale modérément ramifiée de $K$ dans $L$; pour tout $r \in \mathbb{N}$, on note $\mathcal{E}_{r}(L)$ l'ensemble des extension finies $T$ de $L^{\mathrm{mr}}$ contenues dans $L$ et telles que $p^{r}$ divise $\left[T: L^{\mathrm{mr}}\right]$; alors, si $x=\left(x_{T}\right)_{T \in \mathcal{E}_{L / K}}$ est dans $E_{K}(L)$, la famille

$$
\left(x_{T}^{p^{-r}\left[T: L^{m r}\right]}\right)_{T \in \mathcal{E}_{r}(L)}
$$

converge suivant le filtre des sections de $\mathcal{E}_{r}(L)$ vers un élément $j_{K}(x)^{(r)}$ de $C$ pour tout $r \in \mathbb{N}$ et

$$
j_{K}(x)=\left(j_{K}(x)^{(r)}\right)_{r \in \mathbb{N}}
$$

est dans Fr $R$. On montre ensuite que l'application

$$
j_{K}: E_{K}(\bar{K}) \longrightarrow \operatorname{Fr} R
$$

ainsi construite convient et que son image est dense dans $\operatorname{Fr} R$ ( $c f$. [Win, cor. 4.3.4]). On décrira ce plongement d'une autre manière au paragraphe suivant.

\subsubsection{Les anneaux $\mathcal{O}_{\mathcal{E}(K)}$ et $\mathcal{O}_{\mathcal{E}^{\mathrm{nr}} \text {. }}$}

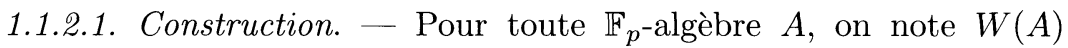
l'anneau des vecteurs de Witt à coefficients dans $A$. On pose

$$
W=W(k)
$$

et on note $K_{0}$ le corps des fractions de $W$. L'anneau $W(\operatorname{Fr} R)$ est muni naturellement d'un Frobenius $\sigma$ et d'une action continue de $G_{K_{0}}$, qui commutent entre eux. Soit

$$
[\epsilon]=\left(\epsilon=\left(\epsilon^{(n)}\right)_{n \in \mathbb{N}}, 0,0, \ldots, 0, \ldots\right)
$$

тОме $126-1998-\mathrm{N}^{\circ} 4$ 
un élément de $W\left(\right.$ Fr $R$ ) tel que $\epsilon^{(0)}=1$ et $\epsilon^{(1)} \neq 1$. Notons $\mathcal{E}_{0}^{\prime}$ le corps des fractions du séparé complété, pour la topologie $p$-adique, de l'anneau

$$
W(([\epsilon]-1)):=W[[[\epsilon]-1]]\left[\frac{1}{[\epsilon]-1}\right] .
$$

Il s'identifie à un sous-corps de $W(\operatorname{Fr} R)$, stable par le Frobenius et l'action de $G_{K_{0}}$ car on a les relations :

$$
\sigma([\epsilon])=[\epsilon]^{p}, \quad \forall g \in G_{K_{0}}, \quad g([\epsilon])=[\epsilon]^{\chi(g)} .
$$

où $\chi: G_{K_{0}} \longrightarrow \mathbb{Z}_{p}^{*}$ désigne le caractère cyclotomique. On en déduit que le groupe $G_{K_{0}}$ opère continûment sur le corps $\mathcal{E}_{0}^{\prime}$ à travers le quotient

$$
\operatorname{Gal}\left(\bigcup_{n \in \mathbb{N}} K_{0}(\sqrt[p]{1}) / K_{0}\right)
$$

qui est isomorphe à un produit de $\mathbb{Z}_{p}$ par un groupe fini $\Gamma_{\text {tor }}$, cyclique d'ordre $p-1$ si $p \neq 2$ (resp. d'ordre 2 si $p=2$ ). On pose

$$
\mathcal{E}_{0}=\left(\mathcal{E}_{0}^{\prime}\right)^{\Gamma_{\text {tor }}}
$$

C'est un corps valué complet dont l'anneau des entiers $\mathcal{O}_{\mathcal{E}_{0}}$ est la complétion $p$-adique de $W\left(\left(\pi_{0}\right)\right)$ où

$$
\pi_{0}=\operatorname{Tr}_{\mathcal{E}_{0}^{\prime} / \mathcal{E}_{0}}([\epsilon]-1)
$$

(cf. [Fo1, A.3.2]). Si $\left(K_{0}\right)_{\infty}$ désigne la $\mathbb{Z}_{p}$-extension cyclotomique de $K_{0}$ contenue dans $\bar{K}$, le corps résiduel $E_{0}$ de $\mathcal{E}_{0}$ est le corps des normes de l'extension $\left(K_{0}\right)_{\infty} / K_{0}$.

On considère alors l'anneau de valuation discrète $\mathcal{O}_{\mathcal{E}^{\text {nr }}}$ qui est la réunion des sous- $\mathcal{O}_{\mathcal{E}_{0}}$-algèbres étales de $W(\operatorname{Fr} R)$ : son corps des fractions $\mathcal{E}^{\text {nr }}$ est la réunion des extensions finies non ramifiées de $\mathcal{E}_{0}$ contenues dans $W(\operatorname{Fr} R)[1 / p]$; son corps résiduel, qui s'identifie à un sous-corps de Fr $R$, est une clôture séparable $E^{\mathrm{sep}}$ de $E_{0}$. Les anneaux $\mathcal{O}_{\mathcal{E}^{\mathrm{nr}}}, \mathcal{E}^{\mathrm{nr}}$ et $E^{\mathrm{sep}}$ sont stables par $\sigma$ et par l'action de $G_{K_{0}}$.

On pose

$$
\mathcal{E}(K)=\left(\mathcal{E}^{\mathrm{nr}}\right)^{\operatorname{Gal}\left(\bar{K} / K_{\infty}\right)}
$$

C'est un corps valué complet, dont l'anneau des entiers est

$$
\mathcal{O}_{\mathcal{E}(K)}=\left(\mathcal{O}_{\mathcal{E}^{\mathrm{nr}}}\right)^{\mathrm{Gal}\left(\bar{K} / K_{\infty}\right)}
$$


l'idéal maximal de $\mathcal{O}_{\mathcal{E}(K)}$ est engendré par $p$; son corps résiduel $E_{K}$ s'identifie au corps des normes de l'extension $K_{\infty} / K$ et est une extension séparable de $E_{0}$ de degré

$$
d_{K}=\left[K_{\infty}: K \cap\left(K_{0}\right)_{\infty}\right] .
$$

Les anneaux $\mathcal{E}(K), \mathcal{O}_{\mathcal{E}(K)}$ et $E_{K}$ sont stables par le Frobenius $\sigma$ et munis d'une action continue de $\Gamma_{K}=\operatorname{Gal}\left(K_{\infty} / K\right)$. De plus, $\Gamma_{K}$ opère trivialement sur le sous-corps $k$ de $E_{K}$, mais ce n'est en général pas le cas pour le corps résiduel $k_{\infty}$.

On pose

$$
G_{E_{K}}=\operatorname{Gal}\left(E^{\mathrm{sep}} / E_{K}\right)=\operatorname{Gal}\left(\mathcal{E}^{\mathrm{nr}} / \mathcal{E}(K)\right) ;
$$

il s'identifie à $\operatorname{Gal}\left(\bar{K} / K_{\infty}\right)$, sous-groupe fermé invariant de $G_{K}$.

Il est important de noter que ces constructions ne dépendent pas du choix de $\epsilon$. De plus, les corps $E_{0}, E_{K}$ et $E^{\text {sep }}$ se plongent de façon naturelle dans Fr $R$ et ils s'identifient alors aux images par l'application $j_{K_{0}}$ de $E_{K_{0}}\left(\left(K_{0}\right)_{\infty}\right), E_{K}\left(K_{\infty}\right)$ et $E_{K}(\bar{K})$.

1.1.2.2. Quelques précisions sur l'action de Galois. - Si $t$ est un relèvement dans $\mathcal{O}_{\mathcal{E}(K)}$ d'une uniformisante de $E_{K}$, alors $\mathcal{O}_{\mathcal{E}(K)}$ s'identifie à la complétion $p$-adique de $W\left(k_{\infty}\right)[[t]][1 / t]$. Mais, en général, il n'est pas possible de choisir $t$ pour que $W\left(k_{\infty}\right)[[t]]$ soit stable à la fois par l'action de $\Gamma_{K}$ et par celle de $\sigma$ : en effet, si c'était le cas, la représentation obtenue en induisant de $K$ à $K_{0}$ la représentation triviale serait de hauteur finie; elle deviendrait donc cristalline sur l'un des $\left(K_{0}\right)^{(n)}$ d'après un résultat de N. Wach $(c f$. [Wac, $\S \mathrm{A} .5]$ ) et cela impliquerait que $K$ est non ramifié sur $\left(K_{0}\right)^{(n)}$ ! Un tel choix est toutefois possible lorsque $d_{K}=\left[k_{\infty}: k\right]$, c'est-à-dire lorsque $K$ est contenu dans une extension non ramifiée de $\left(K_{0}\right)_{\infty}$, auquel cas on peut prendre pour $t$ l'élément $\pi_{0}$ défini plus haut. On peut même préciser un peu plus cela :

Lemme 1.1. - Pour tout $g \in G_{K}$, l'élément $g\left(\pi_{0}\right) / \pi_{0}$ est une unité de l'anneau $W\left[\left[\pi_{0}\right]\right]$. Si l'extension $K_{\infty} / K$ est totalement ramifiée et si $\pi$ est une uniformisante de $E_{K}$, alors pour tout $g \in G_{K}$, l'élément $g(\pi) / \pi$ est une unité fondamentale de $E_{K}$, c'est-à-dire un élément de l'anneau des entiers congru à 1 modulo l'idéal maximal.

Preuve. - Soit $\mathcal{O}_{C}$ le séparé complété pour la topologie $p$-adique de l'anneau des entiers de $\bar{K}$ et soit $\theta: W(R) \rightarrow \mathcal{O}_{C}$ l'homomorphisme de $W$-algèbres introduit par Fontaine ( $c f$. [Fo2]). On voit que le noyau de la restriction de $\theta$ à $W\left[\left[\pi_{0}\right]\right]$ est l'idéal engendré par $\pi_{0}$. Comme $\theta$ est $G_{K^{-}}$ équivariante, pour tout $g \in \Gamma_{K}$, les éléments $\pi_{0}$ et $g\left(\pi_{0}\right)$ engendrent le même idéal de $W\left[\left[\pi_{0}\right]\right]$.

$$
\text { TOME } 126-1998-\mathrm{N}^{\circ} 4
$$


Quant à la deuxième assertion, elle provient simplement du fait que si $K_{\infty} / K$ est totalement ramifiée, le corps résiduel de $E_{K}$ est $k$, sur lequel $G_{K}$ opère trivialement.

1.1.2.3. «Topologie naturelle» sur un $\mathcal{O}_{\mathcal{E}_{0}}$-module de longueur finie. Soient $X$ une indéterminée sur $W$ et $M$ un $W((X))$-module de longueur finie. La topologie naturelle sur $M$ est celle dont une base de voisinages ouverts de 0 est formée par les sous- $W[[X]]$-modules de type fini contenant un système générateur de $M$ sur $W((X))$. Si l'on fixe un tel sous$W[[X]]$-module $N$, alors on peut aussi prendre comme base de voisinages ouverts de 0 pour cette topologie les parties de la forme $X^{n} N$, pour $n$ parcourant $\mathbb{N}$.

Soit $M$ un $\mathcal{O}_{\mathcal{E}_{0}}$-module de longueur finie. Comme $M$ est alors tué par une puissance de $p$, on peut le voir comme un $W\left(\left(\pi_{0}\right)\right)$-module de longueur finie et donc le munir de la topologie naturelle définie plus haut.

\subsection{Lien avec les représentations $\mathbb{Z}_{p}$-adiques de $G_{K}$ : l'équi- valence de catégorie $D_{K}$.}

Rappelons que, si $\Gamma$ est un sous-groupe ouvert non trivial de $\Gamma_{K}$, Fontaine appelle $\Phi$ - $\Gamma$-module sur $\mathcal{O}_{\mathcal{E}(K)}$, la donné d'un $\mathcal{O}_{\mathcal{E}(K)}$-module $M$ muni :

1) d'un opérateur $\sigma$-semi-linéaire, le «Frobenius» $\phi=\phi_{M}: M \rightarrow M$,

2) d'une action $\Gamma$-semi-linéaire continue du groupe $\Gamma$ qui commute avec celle de $\phi$.

On dit que $M$ est un $\Phi-\Gamma$-module étale de torsion si, en tant que $\mathcal{O}_{\mathcal{E}(K)^{-}}$ module, il est de longueur finie et engendré par l'image de $\phi$.

Les $\Phi$ - $\Gamma$-modules étales de torsion $\operatorname{sur} \mathcal{O}_{\mathcal{E}(K)}$ forment une catégorie abélienne, les flèches étant les morphismes $\mathcal{O}_{\mathcal{E}(K)}$-linéaires qui commutent à $\phi$ et à l'action de $\Gamma$. On note

$$
\boldsymbol{\Phi} \Gamma \mathbf{M}_{\mathcal{\mathcal { E } ( K )}}^{\mathrm{et}, p \text {-tor }}
$$

la catégorie des $\Phi-\Gamma_{K}$-modules étales de torsion .

On peut associer à toute représentation $V \in \mathbf{R e p}_{p \text {-tor }}\left(G_{K}\right)$, le $\mathcal{O}_{\mathcal{E}(K)^{-}}$ module :

$$
D_{K}(V)=\left(\mathcal{O}_{\mathcal{E}^{\mathrm{nr}}} \otimes_{\mathbb{Z}_{p}} V\right)^{G_{E_{K}}} .
$$

Il est muni d'une action naturelle de $\Gamma_{K}=G_{K} / G_{E_{K}}$ et on considère

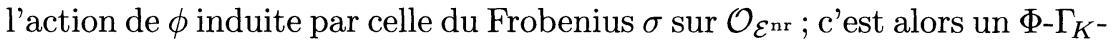
module étale de torsion. 
Inversement, à tout $\Phi-\Gamma_{K}$-module étale de torsion $M$ sur $\mathcal{O}_{\mathcal{E}(K)}$, on peut faire correspondre la représentation de $p$-torsion de $G_{K}$ suivante :

$$
V_{K}(M)=\left(\mathcal{O}_{\mathcal{E}^{\mathrm{nr}}} \otimes_{\mathcal{O}_{\mathcal{E}(K)}} M\right)_{\sigma \otimes \phi_{M}=1} .
$$

Ces deux constructions définissent des foncteurs additifs exacts et Fontaine a montré que l'on a :

ThÉorème 1.2 ( $c f$. [Fo1, A.1.2.4-A.1.2.6]). - Le foncteur $D_{K}$ est une équivalence de catégorie de quasi-inverse $V_{K}$ entre

$$
\boldsymbol{R e p}_{p \text {-tor }}\left(G_{K}\right) \text { et } \boldsymbol{\Phi} \boldsymbol{\Gamma} \mathbf{M}_{\mathcal{E}(K)}^{\text {et, } p \text { tor }} .
$$

De plus, les facteurs invariants sur $\mathbb{Z}_{p}$ d'une représentation de p-torsion $V$ de $G_{K}$ coïncident avec ceux de $D_{K}(V)$ sur $\mathcal{O}_{\mathcal{E}(K)}$ (à des unités près).

\section{Les complexes $C_{\phi, \gamma}$}

On fixe une fois pour toutes un générateur topologique noté $\gamma \mathrm{du}$ groupe $\Gamma_{K}$ et on pose

$$
\tau=\gamma-1, \quad \rho=\phi-1
$$

L'objectif de cette partie est de prouver le résultat suivant qui est à la base de tout cet article :

ThÉorème 2.1. - Soit $V$ une représentation de p-torsion de $G_{K}$. Alors, pour tout entier naturel $i$, le groupe $H^{i}\left(G_{K}, V\right)$ est isomorphe au i-ème groupe de cohomologie du complexe $\mathcal{C}_{\phi, \gamma}\left(D_{K}(V)\right.$ ) (où le premier terme est en degré -1) :

$$
\begin{aligned}
0 \rightarrow D_{K}(V) & \longrightarrow D_{K}(V) \oplus D_{K}(V) \longrightarrow D_{K}(V) \longrightarrow 0 \rightarrow 0 \cdots, \\
x & \longmapsto(\rho(x), \tau(x)) \\
(y, z) & \longmapsto \tau(y)-\rho(z) .
\end{aligned}
$$

En particulier, cela prouve que pour tout $i \geq 3$, les groupes $H^{i}\left(G_{K}, V\right)$ sont nuls. D'où une preuve simple du théorème $\mathrm{A}$.

Remarque. - Si $\gamma^{\prime}$ est un autre générateur topologique de $\Gamma_{K}$, on dispose d'un isomorphisme canonique de complexes

$$
\mathcal{C}_{\phi, \gamma}\left(D_{K}(V)\right) \longrightarrow \mathcal{C}_{\phi, \gamma^{\prime}}\left(D_{K}(V)\right)
$$

l'idéal de $\mathbb{Z}_{p}\left[\left[\Gamma_{K}\right]\right]$ engendré par $\tau^{\prime}=\gamma^{\prime}-1$ est le même que celui engendré par $\tau$; si l'on pose $\tau^{\prime}=\delta \tau$, l'isomorphisme est donné par les applications $x \mapsto x$ en degré $0,(y, z) \mapsto(y, \delta(z))$ en degré 1 et $t \mapsto \delta(t)$ en degré 2 .

L'idée de la démonstration de ce théorème est la suivante : le foncteur $D_{K}$ se prolonge en une équivalence entre la catégorie des $G_{K}$-modules

TOME $126-1998-\mathrm{N}^{\circ} 4$ 
discrets de $p$-torsion et celle, notée $\mathbf{\Phi} \boldsymbol{\Gamma} \mathbf{M}_{\mathcal{O}_{\mathcal{E}}(K)}^{\text {ind-et } p \text {-tor }}$, formée par les limites inductives de $\Phi$ - $\Gamma_{K}$-modules étales de $p$-torsion sur $\mathcal{O}_{\mathcal{E}(K)}$. Ces catégories ont assez d'injectifs, contrairement à $\boldsymbol{\Phi} \boldsymbol{\Gamma} \mathbf{M}_{\mathcal{O}_{\mathcal{E}}(K)}^{\text {et } p \text {-tor }}$. Or calculer la cohomologie des $G_{K}$-modules discrets de $p$-torsion revient à dériver dans la catégorie dont ils sont les objets, le foncteur $H^{0}\left(G_{K}, \cdot\right)$. Mais pour un $G_{K}$-module discret de $p$-torsion $V$, on a :

$$
\left(D_{K}(V)\right)_{\phi=1, \gamma=1} \simeq V^{G_{K}}
$$

Il s'agit donc de dériver dans la catégorie $\mathbf{\Phi} \boldsymbol{\Gamma} \mathbf{M}_{\mathcal{O}_{\mathcal{E}(K)}}^{\text {ind-et, } p \text {-tor }}$ le foncteur qui à un objet $M$ associe le groupe de ses éléments fixes par $\phi$ et $\gamma$.

Rappelons qu'un foncteur $F$ entre deux catégories abéliennes $\mathcal{C}$ et $\mathcal{C}^{\prime}$ est dit effaçable si pour tout objet $M$ de $\mathcal{C}$ et tout $x \in F(M)$ il existe dans $\mathcal{C}$ une injection $u$ de $M$ dans un objet $N$ tel que $F(u)(x)=0$ dans $F(N)$.

On considère alors le foncteur $\mathcal{C}_{\phi, \gamma}$ de $\boldsymbol{\Phi} \boldsymbol{\Gamma} \mathbf{M}_{\mathcal{O}_{\mathcal{E}(K)}}^{\text {ind-et,p-tor }}$ dans la catégorie des complexes de groupes abéliens, qui à un $\Phi$ - $\Gamma_{K}$-module ind-étale $M$ associe le complexe $\mathcal{C}_{\phi, \gamma}(M)$ :

$$
\begin{gathered}
0 \rightarrow M \longrightarrow M \oplus M \longrightarrow M \longrightarrow 0 \rightarrow 0 \cdots, \\
x \longmapsto(\rho(x), \tau(x)), \\
(y, z) \longmapsto \tau(y)-\rho(z) .
\end{gathered}
$$

Ce foncteur est additif, exact et fidèle. Il définit donc un foncteur cohomologique $\left(\mathcal{H}^{i}=H^{i} \circ \mathcal{C}_{\phi, \gamma}, \delta^{i}\right)_{i \in \mathbb{N}}$ de $\boldsymbol{\Phi} \Gamma \mathbf{M}_{\mathcal{O}_{\mathcal{E}(K)}}^{\text {ind-et, } p \text {-tor }}$ dans la catégorie des groupes abéliens.

Comme en degré 0 il coïncide avec la cohomologie galoisienne de $G_{K}$ via $D_{K}$, il suffit de montrer qu'il est effaçable en degré plus élevé (cf. [Poi, cor. p. 65]).

\subsection{Preuve de l'effaçabilité.}

Elle découle du lemme suivant :

LEMme 2.2. - Soient $M$ un objet de $\mathbf{\Phi} \Gamma \mathbf{M}_{\mathcal{O}_{\mathcal{E}(K)}}^{\text {et, } p \text {-tor }}$ et $x$ un élément de $M$. Alors, $M$ se plonge dans un $\Phi-\Gamma_{K}$-module étale de torsion $N_{x}$ tel que $x \in \rho\left(N_{x}\right)$.

Supposons en effet le lemme démontré. Il suffit de vérifier l'effaçabilité du foncteur $\mathcal{H}^{i}$ en degrés 1 et 2 .

Soit $M$ un objet de $\boldsymbol{\Phi} \Gamma \mathbf{M}_{\mathcal{O}_{\mathcal{E}(K)}}^{\text {ind-et,p-tor }}$ : il est réunion de ses sous- $\boldsymbol{\Phi}-\Gamma_{K^{-}}$ modules étales de $p$-torsion. En degré 2 , si $x \in M$, par le lemme 2.2, $M$ 
se plonge dans un objet $N_{x}$ de $\boldsymbol{\Phi} \Gamma \mathbf{M}_{\mathcal{O}_{\mathcal{E}(K)}}^{\text {ind-et, } p \text {-tor }}$ tel que $x \in \rho\left(N_{x}\right)$, et $x$ s'envoie sur 0 dans $\mathcal{H}^{2}\left(N_{x}\right)$.

En degré 1 , soient $y, z \in M$ tels que $\tau(y)=\rho(z)$. Par le lemme 2.2,

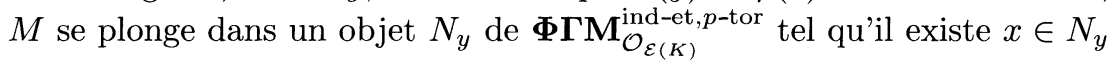
vérifiant $\rho(x)=y$. Soit $s$ un entier tel que

$$
p^{s} x=p^{s} z=0 \text {. }
$$

Notons alors $N_{y, z}$ la somme directe de $N_{y}$ et du $\mathcal{O}_{\mathcal{E}(K)} / p^{s} \mathcal{O}_{\mathcal{E}(K)}$-module libre de rang 1 engendré par un élément $v$ et prolongeons $\phi_{N_{y}}$ et $\gamma$ à $N_{y, z}$ en posant :

$$
\phi(v)=v, \quad \gamma(v)=v+\tau(x)-z .
$$

On vérifie que $\phi(\gamma(v))=\gamma(\phi(v))$ et on voit que le fait que $\tau(v) \in N_{y}$ implique que l'action de $\gamma$ se prolonge en une action semi-linéaire et

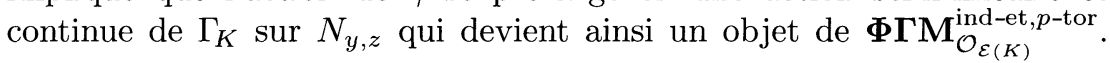
Par construction, on a :

$$
y=\rho(x-v), \quad z=\tau(x-v) .
$$

On en déduit bien que $(y, z)$ s'envoie sur 0 dans le groupe $\mathcal{H}^{1}\left(N_{y, z}\right)$.

Pour prouver le lemme 2.2, il nous faut d'abord étudier de plus près l'action de $\phi$ sur $M$.

\subsection{Réseaux dans des $\Phi-\Gamma_{K}$-modules sur $\mathcal{O}_{\mathcal{E}_{0}}$.}

Définition 2.3. - Soient $X$ une indéterminée et $N$ un $W((X))$-module de longueur finie. On appelle $X$-réseau de $N$ tout sous- $W[[X]]$-module de type fini $\Lambda$ de $N$ qui engendre $N$ en tant que $W((X))$-module.

Remarquons qu'il revient au même de parler de $\mathcal{O}_{\mathcal{E}_{0}}$-module de longueur finie ou de $W\left(\left(\pi_{0}\right)\right)$-module de longueur finie.

Proposition 2.4. - Soit $M$ un $\Phi-\Gamma_{K}$-module sur $\mathcal{O}_{\mathcal{E}_{0}}$, de longueur finie comme $\mathcal{O}_{\mathcal{E}_{0}}$-module. On a les propriétés suivantes :

1) $M$ contient un $\pi_{0}$-réseau stable par $\phi$ et $\Gamma_{K}$, sur lequel $\rho$ est bijective.

2) Pour tout $x \in M$, il existe un entier naturel $r$ et un élément $y$ de $M$ tels que:

$$
\tau^{r}(x)=\rho(y)
$$

Preuve.

1) On commence par construire un $\pi_{0}$-réseau stable par $\phi$ sur lequel $\rho$ est bijectif. On reprend essentiellement la preuve de [Fo1, B1, Lemme 1.4.3].

TOME $126-1998-\mathrm{N}^{\circ} 4$ 
Soient $n \geq 1$ un entier tel que $p^{n}$ annule $M$ et $\left(e_{i}\right)_{1 \leq i \leq d}$ un système générateur de $M$ sur $W\left(\left(\pi_{0}\right)\right)$; il existe alors une matrice $\left(\left(a_{i, j}\right)\right)_{1 \leq i, j \leq d}$ à coefficients dans $W\left(\left(\pi_{0}\right)\right)$ telle que pour tout $i$ dans $\{1, \ldots, d\}$ :

$$
\phi\left(e_{i}\right)=\sum_{1 \leq j \leq d} a_{j, i} e_{j}
$$

Choisissons un entier $s \geq 0$ tel que, pour tous $i, j$ :

$$
\pi_{0}^{s} a_{i, j} \in \pi_{0}^{p^{n-1}} W\left[\left[\pi_{0}\right]\right]+p^{n} W\left(\left(\pi_{0}\right)\right) .
$$

Soit alors $r \in \mathbb{N}$ tel que $p^{n-1}(p-1) r \geq s$. Comme $\sigma\left(\pi_{0}\right) \equiv \pi_{0}^{p}(\bmod p)$, on a $\sigma\left(\pi_{0}^{p^{n-1}}\right) \equiv \pi_{0}^{p^{n}}\left(\bmod p^{n}\right)$. Donc, pour tout $i$ :

$$
\phi\left(\pi_{0}^{p^{n-1} r} e_{i}\right)=\sum_{j=1}^{d}\left(\pi_{0}^{p^{n-1}(p-1) r} a_{j, i}\right)\left(\pi_{0}^{p^{n-1} r} e_{j}\right)
$$

Pour tout $i$, posons

$$
f_{i}=\pi_{0}^{p^{n-1}} e_{i}
$$

et soit $\mathcal{R}$ le sous- $W\left[\left[\pi_{0}\right]\right]$-module de $M$ engendré par les $f_{i}$. D'après la formule précédente, $\mathcal{R}$ est stable par $\phi$. Comme les $f_{i}$ engendrent $M$ sur $W\left(\left(\pi_{0}\right)\right)$, on voit que $\mathcal{R}$ est un $\pi_{0}$-réseau de $M$ stable par $\phi$.

On va maintenant montrer que :

$$
\forall x \in \mathcal{R}, \quad \lim _{r \rightarrow+\infty} \phi^{r}(x)=0 .
$$

Si $x \in \mathcal{R}$ s'écrit

$$
x=\sum_{1 \leq i \leq d} x_{i} f_{i}
$$

on a :

$$
\phi(x)=\sum_{j=1}^{d}\left(\sum_{i=1}^{d} \sigma\left(x_{i}\right)\left(\pi_{0}^{p^{n-1}(p-1) r} a_{j, i}\right)\right) f_{j} .
$$

On en déduit que $\phi(x) \in \pi_{0}^{p^{n-1}} \mathcal{R}$. De même, si $t \in \mathbb{N}$ et si $y \in \pi_{0}^{t p^{n-1}} \mathcal{R}$, alors $\phi(y) \in \pi_{0}^{t p^{n}} \phi(\mathcal{R}) \subset \pi_{0}^{(1+t p) p^{n-1}} \mathcal{R}$. Donc :

$$
\lim _{r \rightarrow+\infty} \phi^{r}(x)=0 \text {. }
$$

Par conséquent, $\sum_{n \in \mathbb{N}}\left(-\phi^{n}\right)(x)$ converge dans $\mathcal{R}$. L'application $\rho$ est donc inversible sur $\mathcal{R}$ et on a :

$$
\rho^{-1}=\sum_{r \in \mathbb{N}}\left(-\phi^{r}\right)
$$

Le fait que $M$ soit un $W\left(\left(\pi_{0}\right)\right)$-module de longueur finie implique que $\mathcal{R}$ est un voisinage ouvert de 0 dans $M$. 
Soit $x \in \mathcal{R}$. La continuité de l'action de $\Gamma_{K}$ sur $M$ implique que la suite des $\gamma^{p^{r}}(x)-x$ tend vers 0 dans $M$ donc que $\gamma^{p^{r}}(x)-x$ et aussi $\gamma^{p^{r}}(x)$ sont dans $\mathcal{R}$ pour $\mathrm{r}$ assez grand. Soient $x_{1}, x_{2}, \ldots, x_{d}$ des générateurs de $\mathcal{R}$ sur $W\left[\left[\pi_{0}\right]\right]$ et soit $r$ un entier $\geq 1$ tel que les $\gamma^{p^{r}}\left(x_{i}\right)$ sont dans $\mathcal{R}$. En utilisant le fait que l'anneau $W\left[\left[\pi_{0}\right]\right]$ est stable par $\Gamma_{K}$, on voit que

$$
\mathcal{R}^{\prime}=\sum_{0 \leq t<p^{r}} \gamma^{t}(\mathcal{R})
$$

l'est aussi. C'est encore un $\pi_{0}$-réseau de $M$ stable par $\phi$. De plus, pour tout $x \in \mathcal{R}^{\prime}$, la suite des $\phi^{r}(x)$ tend vers 0 , donc $\rho$ est encore inversible sur $\mathcal{R}^{\prime}$.

2) Comme le réseau $\mathcal{R}^{\prime}$ que l'on vient de construire est un voisinage ouvert de 0 dans $M$ sur lequel $\rho$ est inversible, il suffit de prouver que, pour tout $x \in M$, la suite des $\tau^{r}(x)$ tend vers 0 lorsque $\mathrm{r}$ tend vers $+\infty$, ce qui résulte de la continuité de l'action de $\Gamma_{K}$ sur $M$.

\subsection{Preuve du lemme 2.2.}

Soient $M$ un objet de $\boldsymbol{\Phi} \boldsymbol{\Gamma} \mathbf{M}_{\mathcal{O}_{\mathcal{E}(K)}}^{\text {et, } p \text { tor }}$ et $x$ un élément de $M$. Comme $\mathcal{O}_{\mathcal{E}(K)}$ est un $\mathcal{O}_{\mathcal{E}_{0}}$-module de type fini et $\Gamma_{K}$ un sous-groupe ouvert de $\Gamma_{K_{0}}$, on peut voir $M$ comme un $\Phi$ - $\Gamma_{K}$-module de torsion sur $\mathcal{O}_{\mathcal{E}_{0}}$. D'après la proposition 2.4, on peut trouver un entier naturel $r$ tel que

$$
(\gamma-1)^{r}(x) \in \rho(M) .
$$

Si $r=0$, il n'y a rien à prouver. Si $r \geq 1$, on choisit $t_{0} \in M$ tel que $\tau^{r}(x)=\rho\left(t_{0}\right)$. Soit $n$ un entier tel que $p^{n}$ annule $M$. On considère alors la somme directe $N_{x}$ de $M$ et du $\mathcal{O}_{\mathcal{E}(K)} / p^{n} \mathcal{O}_{\mathcal{E}(K)}$-module libre de rang $r$ engendré par des éléments $t_{1}, \ldots, t_{r}$. On prolonge l'action de $\phi$ et de $\gamma$ à $N_{x}$ en posant pour tout $i \in\{1, \ldots, r\}$ :

$$
\phi\left(t_{i}\right)=t_{i}+\tau^{r-i}(x), \quad \gamma\left(t_{i}\right)=t_{i}+t_{i-1} .
$$

Par construction, on a

$$
\rho\left(t_{r}\right)=x
$$

et $\phi$ commute avec $\gamma$ : en effet, comme c'est le cas sur $M$, il suffit, par semi-linéarité, de remarquer que pour tout $i \in\{1, \ldots, r\}$, on a :

$$
\begin{aligned}
\phi\left(\gamma\left(t_{i}\right)\right) & =\phi\left(t_{i}\right)+\phi\left(t_{i-1}\right) \\
& =t_{i}+t_{i-1}+\tau\left(\tau^{r-i}(x)\right)+\tau^{r-i}(x) \\
& =\gamma\left(t_{i}+\tau^{r-i}(x)\right)=\gamma\left(\phi\left(t_{i}\right)\right) .
\end{aligned}
$$

TOME $126-1998-\mathrm{N}^{\circ} 4$ 
De plus, il résulte facilement de ce que $\tau^{r}\left(t_{i}\right) \in M$ pour tout $i$ que l'action de $\gamma$ se prolonge en une action linéaire et continue de $\Gamma_{K}$ sur $N_{x}$.

Le $\mathcal{O}_{\mathcal{E}(K)}$-module de longueur finie $N_{x}$ est ainsi muni d'une structure de $\Phi-\Gamma_{K}$-module sur $\mathcal{O}_{\mathcal{E}(K)}$. Le fait qu'il est étale se déduit immédiatement du fait que $M$ l'est.

\section{L'opérateur $\psi$}

À la différence du théorème $\mathrm{A}$, le complexe $\mathcal{C}_{\phi, \gamma}\left(D_{K}(V)\right)$ ne semble pas donner une preuve directe simple du théorème B. Dans cette partie, on introduit un inverse à gauche additif $\psi$ du Frobenius $\phi$ de $D_{K}(V)$; il permettra au paragraphe suivant de donner un complexe quasi-isomorphe à $\mathcal{C}_{\phi, \gamma}\left(D_{K}(V)\right)$, dont on pourra dévisser de façon naturelle la cohomologie pour prouver le théorème $\mathrm{B}$. Dans cette décomposition, la structure du noyau de $\psi$ joue un rôle fondamental et constitue le résultat essentiel de cette partie (th. 3.8).

\subsection{Définition.}

3.1.1. Cas de l'anneau $\mathcal{O}_{\mathcal{E}(K)}$. - L'extension $\mathcal{E}^{\mathrm{nr}} / \sigma\left(\mathcal{E}^{\mathrm{nr}}\right)$ est de degré $p$, de même que l'extension résiduelle qui est purement inséparable. Ceci implique que l'on a l'inclusion :

$$
\operatorname{Tr}_{\mathcal{E}^{\mathrm{nr}} / \sigma\left(\mathcal{E}^{\mathrm{nr}}\right)}\left(\mathcal{O}_{\mathcal{E}^{\mathrm{nr}}}\right) \subset p \sigma\left(\mathcal{O}_{\mathcal{E}^{\mathrm{nr}}}\right)
$$

Comme $\sigma$ est injectif, on peut alors définir une application

$$
\psi=\psi_{\mathcal{O}_{\mathcal{E}^{\mathrm{nr}}}}: \mathcal{O}_{\mathcal{E}^{\mathrm{nr}}} \longrightarrow \mathcal{O}_{\mathcal{E}^{\mathrm{nr}}}
$$

en posant pour tout $x \in \mathcal{O}_{\mathcal{E}^{\text {nr }}}$ :

$$
\psi(x)=\frac{1}{p} \sigma^{-1}\left(\operatorname{Tr}_{\mathcal{E}^{\mathrm{nr}} / \sigma\left(\mathcal{E}^{\mathrm{nr}}\right)}(x)\right) .
$$

C'est un homomorphisme de groupe, $G_{K}$-équivariant, qui vérifie, pour tout $a$ et tout $b$ dans $\mathcal{O}_{\mathcal{E}^{\text {nr }}}$ :

$$
\psi(a \sigma(b))=b \psi(a) \quad \text { et } \quad \psi(1)=1 .
$$

Il induit une application

$$
\psi=\psi_{\mathcal{O}_{\mathcal{E}(K)}}: \mathcal{O}_{\mathcal{E}(K)} \longrightarrow \mathcal{O}_{\mathcal{E}(K)}
$$

commutant avec l'action de $\Gamma_{K}$, par restriction aux éléments fixes $\operatorname{par} G_{E_{K}}$. 


\subsubsection{Cas général.}

Proposition 3.1. - Soit $M$ un $\Phi-\Gamma_{K}$-module étale de torsion sur $\mathcal{O}_{\mathcal{E}(K)}$. Il existe une unique application additive

$$
\psi=\psi_{M}: M \longrightarrow M
$$

vérifiant

$$
\psi_{M}\left(a \phi_{M}(x)\right)=\psi_{\mathcal{O}_{\mathcal{E}(K)}}(a) x, \quad \forall a \in \mathcal{O}_{\mathcal{E}(K)}, x \in M .
$$

Cette application est surjective et vérifie $\psi_{M} \circ \phi_{M}=\mathrm{id}_{M}$ et

$$
\psi_{M}(\sigma(a) x)=a \psi_{M}(x), \quad \forall a \in \mathcal{O}_{\mathcal{E}(K)}, x \in M .
$$

Preuve. - L'unicité résulte de ce que, comme $M$ est étale, il est engendré, comme $\mathcal{O}_{\mathcal{E}(K)}$-module par l'image de $\phi_{M}$.

On peut vérifier l'existence ainsi : l'équivalence de catégorie $V_{K}$ (th. 1.2) associe à $M$ une représentation de $p$-torsion $V$, qui nous permet d'identifier $M$ à $D_{K}(V)$ et $\phi_{M}$ à la restriction de $\sigma \otimes \mathrm{id}_{V}$. Via $D_{K} \circ V_{K}$, on constate que l'application $\psi_{M}: M \rightarrow M$ induite par $\psi_{\mathcal{O}_{\mathcal{E}^{\mathrm{nr}}}} \otimes \mathrm{id}_{V}$ convient. Le reste de la proposition est évident.

\subsection{Premières propriétés.}

Dans toute la suite $d u \S 3, n$ est un entier $\geq 1, M$ est un $\Phi-\Gamma_{K}$-module étale sur $\mathcal{O}_{\mathcal{E}(K)}$, tué par $p^{n}$ et $V=V_{K}(M)$.

On pose

$$
\ell_{V}=\operatorname{long}_{\mathbb{Z}_{p}}(V)
$$

Alors $M$ est un $\mathcal{O}_{\mathcal{E}(K)}$-module de longueur $\ell_{V}$; c'est donc aussi un $\mathcal{O}_{\mathcal{E}_{0}}$-module de longueur $d_{K} \ell_{V}$; comme $\mathcal{O}_{\mathcal{E}_{0}}$ est la complétion $p$-adique de $W\left(\left(\pi_{0}\right)\right)$ et comme $M$ est de $p$-torsion, $M$ est encore un $W\left(\left(\pi_{0}\right)\right)$ module de longueur $d_{K} \ell_{V}$ (cf. 1.1.2).

Proposition 3.2. - On a les propriétés suivantes :

1) L'application $\psi$ est continue.

2) Pour tout entier $r \geq 1, \operatorname{Ker}\left(\psi^{r}\right)$ est un sous- $\sigma^{r}\left(\mathcal{O}_{\mathcal{E}(K)}\right)$-module de $M$ de longueur finie égale $\grave{a}\left(p^{r}-1\right) \ell_{V}$ (donc aussi un $W\left(\left(\sigma^{r}\left(\pi_{0}\right)\right)\right)$-module de longueur $\left.\left(p^{r}-1\right) d_{K} \ell_{V}\right)$, stable par $\Gamma_{K}$ et:

$$
M=\operatorname{Ker}\left(\psi^{r}\right) \oplus \phi^{r}(M), \quad \operatorname{Ker}\left(\psi^{r}\right)=\bigoplus_{i=0}^{r-1} \phi^{i}(\operatorname{Ker} \psi) .
$$

De plus, l'application de $(\operatorname{Ker} \psi)^{r}$ dans $\operatorname{Ker}\left(\psi^{r}\right)$ qui à la famille $\left(a_{i}\right)_{0 \leq i \leq r-1}$ associe $\sum_{i=0}^{r-1} \phi^{i}\left(a_{i}\right)$ est un isomorphisme de groupes $\Gamma_{K}$-équivariant.

TOME $126-1998-\mathrm{N}^{\circ} 4$ 
3) Pour tout $x \in M, \psi(x)$ est l'unique élément $y$ de $M$ tel que $x-\phi(y) \in \operatorname{Ker} \psi$.

4) L'application naturelle

$$
\operatorname{Ker} \psi \longrightarrow M / \phi(M)
$$

est un homéomorphisme $\sigma\left(\mathcal{O}_{\mathcal{E}(K)}\right)$-linéaire et $\Gamma_{K}$-équivariant.

5) $S i$ on note $M_{\text {nil }}$ l'ensemble des éléments $\psi$-nilpotents de $M$, l'application de $\bigoplus_{i \in \mathbb{N}} \operatorname{Ker} \psi$ dans $M_{\text {nil }}$ qui à la suite presque nulle $\left(a_{i}\right)_{i \in \mathbb{N}}$ associe $\sum_{i \in \mathbb{N}} \phi^{i}\left(a_{i}\right)$ est un isomorphisme de groupes qui commute avec l'action de $\Gamma_{K}$.

Preuve. - L'assertion 1) est immédiate sur la définition de $\psi$. Montrons le 2). On déduit, par récurrence sur $r$ du (2) de la proposition 3.1 que, pour tout entier $r \geq 1$, si $a \in \mathcal{O}_{\mathcal{E}(K)}$ et $x \in M$, alors $\psi^{r}\left(\sigma^{r}(a) x\right)=a \psi^{r}(x)$ et $\operatorname{Ker}\left(\psi^{r}\right)$ est bien un sous- $\sigma^{r}\left(\mathcal{O}_{\mathcal{E}(K)}\right)$-module de $M$. Comme $\mathcal{O}_{\mathcal{E}(K)}$ est fini sur $\sigma^{r}\left(\mathcal{O}_{\mathcal{E}(K)}\right)$, M est un $\sigma^{r}\left(\mathcal{O}_{\mathcal{E}(K)}\right)$-module de longueur finie et Ker $\psi^{r}$ aussi. Donc

Pour tout $x \in M$, et tout entier naturel $r$, on a $x-\phi^{r}\left(\psi^{r}(x)\right) \in \operatorname{Ker}\left(\psi^{r}\right)$.

$$
M=\phi^{r}(M)+\operatorname{Ker}\left(\psi^{r}\right) .
$$

De plus, si $\phi^{r}(x)$ appartient à $\operatorname{Ker}\left(\psi^{r}\right)$, alors $x=\psi^{r}\left(\phi^{r}(x)\right)=0$, donc $\phi^{r}(x)=0$ et la somme est directe.

En particulier, tout $x$ dans $M$ s'écrit (de manière unique) sous la forme $x=\phi(y)+z$ avec $y, z$ dans $M$ et $\psi(z)=0$, et on a donc $\psi^{r}(x)=\psi^{r-1}(y)$ pour tout $r \geq 1$. On en déduit alors par récurrence sur $r$ que :

$$
\forall r \geq 1, \quad \operatorname{Ker}\left(\psi^{r}\right)=\sum_{i=0}^{r-1} \phi^{i}(\operatorname{Ker} \psi)
$$

Mais cette somme est directe : en effet, soit $m$ un entier compris entre 0 et $(r-1)$; alors l'égalité $\sum_{i=0}^{r-1} \phi^{i}\left(x_{i}\right)=0$ pour des éléments $x_{i}$ dans Ker $\psi$ entraîne, en appliquant $\psi^{m}$ :

$$
x_{m}=-\sum_{i=m+1}^{r-1} \phi^{i-m}\left(x_{i}\right) \quad \text { si } \quad m \leq r-2, \quad x_{r-1}=0 .
$$

Dans tous les cas, $x_{m} \in \phi(M) \cap \operatorname{Ker} \psi$ et est donc nul ainsi que $\phi^{m}\left(x_{m}\right)$. On en déduit que pour tout entier $r \geq 1$, l'application de $(\operatorname{Ker} \psi)^{r}$ vers $\operatorname{Ker}\left(\psi^{r}\right)$ qui à $\left(x_{i}\right)_{0 \leq i \leq r-1}$ associe $\sum_{i=0}^{r-1} \phi^{i}\left(x_{i}\right)$ est une bijection. Elle est de plus clairement additive. 
Enfin, on a

$$
\begin{gathered}
\operatorname{long}_{\sigma^{r}\left(\mathcal{O}_{\mathcal{E}(K)}\right)}(M)=p^{r} \operatorname{long}_{\mathcal{O}_{\mathcal{E}(K)}}(M), \\
\operatorname{long}_{\sigma^{r}\left(\mathcal{O}_{\mathcal{E}(K)}\right)}\left(\phi^{r}(M)\right)=\operatorname{long}_{\mathcal{O}_{\mathcal{E}(K)}}(M) .
\end{gathered}
$$

Comme $M=\operatorname{Ker}\left(\psi^{r}\right) \oplus \phi^{r}(M)$, on obtient

$$
\begin{aligned}
\operatorname{long}_{\sigma^{r}\left(\mathcal{O}_{\mathcal{E}(K)}\right)}(\operatorname{Ker} \psi) & =\operatorname{long}_{\sigma^{r}\left(\mathcal{O}_{\mathcal{E}(K)}\right)}(M)-\operatorname{long}_{\sigma^{r}\left(\mathcal{O}_{\mathcal{E}(K)}\right)}\left(\phi^{r}(M)\right) \\
& =\left(p^{r}-1\right) \operatorname{long}_{\mathcal{O}_{\mathcal{E}(K)}}(M)=\left(p^{r}-1\right) \ell_{V}
\end{aligned}
$$

D'où le 2).

Les trois autres assertions s'en déduisent aisément.

Proposition 3.3. - Le $W\left(\left(\pi_{0}\right)\right)$-module de type fini $M$ contient un $\pi_{0}$-réseau $\mathcal{N}$ stable par $\psi$ et $\Gamma_{K}$ tel que :

$$
\psi(\mathcal{N})=\mathcal{N} \quad \text { et } \quad M=\mathcal{N}+M_{\text {nil }}
$$

Commençons par établir un lemme :

Lemme 3.4. - Soit $N$ un sous-W $\left[\left[\pi_{0}\right]\right]-$ module de $M$. Alors :

1) $\psi(N)$ est un sous- $W\left[\left[\pi_{0}\right]\right]$-module de $M$.

2) Si $N$ est de type fini sur $W\left[\left[\pi_{0}\right]\right], \psi(N)$ l'est aussi.

3) Posons $q_{n}=\pi_{0}^{p^{n-1}}$. Si $N$ est stable par $\phi$, on $a$ :

$$
\forall r \in \mathbb{N}, \forall i \in \mathbb{Z}, \quad \psi^{r}\left(q_{n}^{i} N\right) \supset q_{n}^{\sup (0, i)} N .
$$

Preuve.

1) Pour tout $x \in N$ et tout $\lambda \in W\left[\left[\pi_{0}\right]\right]$, on a $\lambda \psi(x)=\psi(\sigma(\lambda) x)$. Comme $\psi$ est additive, $\psi(N)$ est bien un sous- $W\left[\left[\pi_{0}\right]\right]$-module de $M$.

2) Comme $W\left[\left[\pi_{0}\right]\right]$ est fini sur $W\left[\left[\sigma\left(\pi_{0}\right)\right]\right]$, si $N$ est de type fini sur $W\left[\left[\pi_{0}\right]\right]$, il l'est aussi sur $W\left[\left[\sigma\left(\pi_{0}\right)\right]\right]$ donc $\psi(N)$ est de type fini sur $\sigma^{-1}\left(W\left[\left[\sigma\left(\pi_{0}\right)\right]\right]\right)=W\left[\left[\pi_{0}\right]\right]$.

3) Pour tout $x \in N$ et tout $r \in \mathbb{N}, \psi^{r}\left(\phi^{r}(x)\right)=x$, donc $\psi^{r}(N)$ contient $N$. Soit $i$ un entier relatif :

- si $i$ est négatif : $\psi^{r}\left(q_{n}^{i} N\right)$ contient $\psi^{r}(N)$ et donc $N$ d'après ce qui précède;

- si $i$ est positif : comme $\sigma\left(\pi_{0}\right) \equiv \pi_{0}^{p} \bmod p, \phi\left(q_{n}\right) \equiv q_{n}^{p}\left(\bmod p^{n}\right)$, donc $\phi\left(q_{n}^{i} x\right)=\left(q_{n}^{i}\right)^{p} \phi(x)$, pour tout $x \in N$. Donc $q_{n}^{i} N$ est stable par $\phi$ et $\psi^{r}\left(q_{n}^{i} N\right)$ contient $q_{n}^{i} N$.

$$
\text { TOME } 126-1998-\mathrm{N}^{\circ} 4
$$


Ce lemme implique :

Corollaire 3.5. - Soit $N$ un $\pi_{0}$-réseau de $M$ stable par $\phi$. Alors pour tout entier relatif $i$ et tout entier naturel $r, \psi^{r}\left(q_{n}^{i} N\right)$ est un $\pi_{0}$-réseau de $M$.

Preuve de la proposition 3.3. - On va procéder en deux étapes : on construit d'abord un $\pi_{0}$-réseau $\mathcal{N}_{0}$ stable par $\psi$ et $\Gamma_{K}$ et sur lequel $\psi$ est surjective; puis on élargi $\mathcal{N}_{0}$ de sorte qu'il vérifie la seconde condition.

1) Par la proposition 2.4 , on sait que $M$ contient un $\pi_{0}$-réseau $N$ stable par $\phi$ et $\Gamma_{K}$. Pour tout $r \in \mathbb{N}$, on a $\psi^{r}(N) \subset \psi^{r+1}(N)$ et le corollaire 3.5 nous montre alors que $\left(\psi^{r}(N)\right)_{r \in \mathbb{N}}$ est une suite croissante de $\pi_{0}$-réseaux de $M$. On va prouver qu'elle est stationnaire et on pourra donc prendre pour $\mathcal{N}_{0}$ la limite de cette suite (qui sera bien stable par $\Gamma_{K}$ puisque $N$ l'est, et $\psi$ et $\Gamma_{K}$ commutent).

Rappelons qu'on a choisi $n \geq 1$ tel que $p^{n}$ annule $M$. Toujours grâce au corollaire 3.5, $\psi\left(q_{n}^{-i} N\right)$ est un $\pi_{0}$-réseau pour tout entier $i$ et il existe donc un entier $s$ tel que $q_{n}^{-s} N$ contienne les $\pi_{0}$-réseaux $\psi\left(q_{n}^{-i} N\right)$ pour $i \in\{0, \ldots, p-1\}$. Considérons alors la suite d'entiers définie par :

$$
s_{1}=s, \quad s_{r+1}=s+t_{r},
$$

où $t_{r}$ est la partie entière de $s_{r} / p$. On va prouver par récurrence que pour tout entier $r \geq 1, \psi^{r}(N)$ est contenu dans le $\pi_{0}$-réseau $q_{n}^{-s_{r}} N$ : c'est clair pour $r=1$; supposons alors que ce soit vrai pour un entier $r \geq 1$. Alors, par division euclidienne, $s_{r}=p t_{r}+i_{r}$, où $i_{r}$ est un entier compris entre 0 et $(p-1)$, et $\psi^{r+1}(N)$ est contenu dans $\psi\left(q_{n}^{-s_{r}} N\right)=q_{n}^{-t_{r}} \psi\left(q_{n}^{-i_{r}} N\right)$, donc, par choix de $s$, dans $q_{n}^{-t_{r}-s} N=q_{n}^{-s_{r+1}} N$. Ce qui achève la récurrence.

Or la suite $\left(s_{r}\right)_{r \geq 1}$ est majorée par la partie entière de $s p /(p-1)$ que l'on notera $\ell$. On en déduit que pout tout $r, \psi^{r}(N)$ est contenu dans le $\pi_{0}$-réseau $q_{n}^{-\ell} N$ qui est un $W\left[\left[\pi_{0}\right]\right]$-module noethérien, donc la suite croissante $\psi^{r}(N)$ est bien stationnaire.

2) Construisons maintenant $\mathcal{N}$. Donnons-nous un $\pi_{0}$-réseau $\mathcal{N}_{0}$ de $M$ stable par $\psi$ et $\Gamma_{K}$ et tel que $\psi\left(\mathcal{N}_{0}\right)=\mathcal{N}_{0}$.

Le lemme 1.1 entraîne que pour tout $g \in \Gamma_{k}$, les éléments $q_{n}=\pi_{0}^{p^{n-1}}$ et $g\left(q_{n}\right)$ engendrent le même idéal de $W\left[\left[\pi_{0}\right]\right]$; par conséquent le $\pi_{0}$-réseau $q_{n}^{-1} \mathcal{N}_{0}$ est stable par $\Gamma_{K}$. Comme

$$
\psi\left(q_{n}^{-1} \mathcal{N}_{0}\right) \subset \psi\left(q_{n}^{-p} \mathcal{N}_{0}\right)=q_{n}^{-1} \psi\left(\mathcal{N}_{0}\right)=q_{n}^{-1} \mathcal{N}_{0}
$$

il l'est aussi par $\psi$. 
Par le corollaire 3.5, $\left(\psi^{r}\left(q_{n}^{-1} \mathcal{N}_{0}\right)\right)_{r \in \mathbb{N}}$ est une suite décroissante de $\pi_{0^{-}}$ réseaux de $M$ contenant tous $\mathcal{N}_{0}\left(=\psi^{r}\left(\mathcal{N}_{0}\right)\right.$ pour tout entier naturel $\left.r\right)$. Cette suite est donc stationnaire et on note $\mathcal{N}$ sa limite. Il est clair que $\mathcal{N}$ est un $\pi_{0}$-réseau de $M$ stable par $\psi$ et $\Gamma_{K}$ et que l'on a $\psi(\mathcal{N})=\mathcal{N}$.

Enfin, soit $x \in M$. Choisissons un entier naturel $r$ tel que $y=q_{n}^{p^{r}} x$ soit dans $\mathcal{N}_{0}$. L'élément $\psi^{r}(x)$ appartient alors à $q_{n}^{-1} \mathcal{N}_{0}$, et par construction de $\mathcal{N}$, il existe $r^{\prime}$ tel que $\psi^{r^{\prime}}(x) \in \mathcal{N}$. Comme $\psi$ est surjective sur $\mathcal{N}$, il existe $z \in \mathcal{N}$ tel que $\psi^{r^{\prime}}(x)=\psi^{r^{\prime}}(z)$. On a alors $x=z+(x-z)$ avec $z \in \mathcal{N}$ et $(x-z) \in M_{\text {nil }}$.

Proposition 3.6. - On a les propriétés suivantes:

1) Soit $M^{\prime}$ le plus grand sous- $W$-module de $M$ contenu dans $(\psi-1)(M)$. Le $W$-module $M / M^{\prime}$ est de longueur finie.

2) Si $K$ est une extension finie de $\mathbb{Q}_{p}$, le groupe $M /(\psi-1)(M)$ est fini.

Preuve. - Soit $\mathcal{R}$ un $\pi_{0}$-réseau de $M$ stable par $\phi$ sur lequel $\phi-1$ est bijectif ( $c f$. prop. 2.4). On a

$$
(\psi-1)(M) \supset(\psi-1)(\phi(M))=(\phi-1)(M) \supset(\phi-1)(\mathcal{R})=\mathcal{R} .
$$

Comme $\psi-1$ est bijectif sur $M_{\text {nil }}$, on a aussi $(\psi-1)(M) \supset M_{\text {nil }}$; de ce fait,

$$
(\psi-1)(M) \supset \mathcal{R}+M_{\text {nil }}
$$

et, pour vérifier 1), il suffit de montrer que, si $M^{\prime \prime}=\mathcal{R}+M_{\text {nil }}$, alors le $W$-module $M / M^{\prime \prime}$ est de longueur finie.

Soit $\mathcal{N}$ comme dans la proposition 3.3. On voit que

$$
M / M^{\prime \prime}=\left(\mathcal{N}+M_{\text {nil }}\right) /\left(\mathcal{R}+M_{\text {nil }}\right)
$$

s'identifie à un quotient de $\mathcal{N} / \mathcal{N} \cap \mathcal{R}$. Ce dernier $W$-module est de longueur finie comme quotient d'un $\pi_{0}$-réseau $\mathcal{N}$ de $M$ par un autre $\pi_{0^{-}}$ réseau contenu dans $\mathcal{N}$.

Enfin, si $\left[K: \mathbb{Q}_{p}\right]$ est fini, $k$ est fini, donc $W$ est fini sur $\mathbb{Z}_{p}$ et tout $W$ module de longueur finie est un groupe fini. Donc $M / M^{\prime}$ et son quotient $M /(\psi-1)(M)$ sont des groupes finis.

\subsection{Le théorème de structure.}

\subsubsection{Un résultat auxiliaire. - Posons}

$$
\pi_{1}=\sigma\left(\pi_{0}\right) .
$$

Soit $N$ un $W\left(\left(\pi_{1}\right)\right)$-module de longueur finie muni d'une action $\Gamma_{K}$-semilinéaire et continue de $\Gamma_{K}$. La continuité de cette action implique que, 
pour tout $x \in N$, la suite des $\tau^{r}(x)$ tend vers 0 . Comme l'action de $\tau$ est $W$-linéaire, $N$ a une structure naturelle de $W[[\tau]]$-module et tout sous$W\left[\left[\pi_{1}\right]\right]$-module de $N$ stable par $\Gamma_{K}$ (en particulier tout $\pi_{1}$-réseau) est aussi un sous- $W[[\tau]]$-module.

Proposition 3.7. - Soit $N$ un $W\left(\left(\pi_{1}\right)\right)$-module de longueur finie muni d'une action semi-linéaire et continue de $\Gamma_{K}$. On suppose que l'action de $\tau$ est inversible sur $N$, que $N$ est aussi un $W((\tau))$-module de longueur finie et qu'il existe un $\pi_{1}$-réseau de $N$ stable par $\Gamma_{K}$ qui est aussi un $\tau$-réseau. Alors :

1) Tout $\pi_{1}$-réseau de $N$ stable par $\Gamma_{K}$ est encore un $\tau$-réseau.

2) Pour tout $\pi_{1}$-réseau $S$ de $N$, il existe des $\pi_{1}$-réseaux $S_{1}$ et $S_{2}$ de $N$ stable par $\Gamma_{K}$ tels que $S_{1} \subset S \subset S_{2}$.

3) Pour tout $\tau$-réseau $T$ de $N$, il existe des $\pi_{1}$-réseaux $T_{1}$ et $T_{2}$ de $N$ stable par $\Gamma_{K}$ tels que $T_{1} \subset T \subset T_{2}$.

Preuve.

1) Soient $S_{0}$ et $S$ deux $\pi_{1}$-réseaux de $N$ stables par $\Gamma_{K}$. Supposons que $S_{0}$ soit un $\tau$-réseau et montrons que $S$ en est aussi un :

a) Si $S_{0} \subset S$, alors $S$ contient un $\tau$-réseau. En outre $\Gamma_{K}$ opère linéairement sur le $W$-module de longueur finie $S / S_{0}$; on en déduit que $\tau^{r}$ opère trivialement sur ce quotient pour $r$ assez grand donc que $\tau^{r} S \subset S_{0}$, ou encore que $S$ est contenu dans le $\tau$-réseau $\tau^{-r} S_{0}$. On en déduit que $S$ est bien un $\tau$-réseau.

b) Si $S \subset S_{0}$, alors $S$ est contenu dans un $\tau$-réseau. Comme $\Gamma_{K}$ opère linéairement sur le $W$-module de longueur finie $S_{0} / S$, on voit que $\tau^{s}$ agit trivialement sur $S_{0} / S$ pour $s$ assez grand. Donc $S$, qui contient le $\tau$-réseau $\tau^{s} S_{0}$, en est un aussi.

c) Dans le cas général, $S_{0}+S$ est un $\tau$-réseau grâce à (a), et (b) montre donc que $S$ aussi.

2) Si $S_{0}$ est comme ci-dessus et si $S$ est un $\pi_{1}$-réseau de $N$, il existe des entiers $r, s \in \mathbb{Z}$ tels que $\pi_{1}^{s} S_{0} \subset S \subset \pi_{1}^{r} S_{0}$ et l'assertion résulte de ce que, comme l'idéal de $W\left[\left[\pi_{1}\right]\right]$ engendré par $\pi_{1}$ est stable par $\Gamma_{K}$ (cf. lemme 1.1), les $\pi_{1}$-réseaux $\pi_{1}^{s} S_{0}$ et $\pi_{1}^{r} S_{0}$ le sont aussi.

3) Remarquons d'abord que, pour tout $i \in \mathbb{N}$, il existe $r \in \mathbb{N}$ tel que $\pi_{1}^{r} S_{0} \subset \tau^{i} S_{0}$ : en effet la suite des $\pi_{1}^{r} S_{0}+\tau^{i} S_{0}$, pour $r \in \mathbb{N}$, est une suite décroissante de sous- $W$-modules de $S_{0}$ contenant $\tau^{i} S_{0}$. Comme $S_{0} / \tau^{i} S_{0}$ est un $W$-module de longueur finie, il existe $r$ tel que $\pi_{1}^{r} S_{0}+\tau^{i} S_{0}=\pi_{1}^{r+1} S_{0}+\tau^{i} S_{0}$ et, comme $S_{0}$ est séparé et complet pour la topologie $\pi_{1}$-adique, on en déduit que $\pi_{1}^{r} S_{0} \subset \tau^{i} S_{0}$. 
De même, pour tout $j \in \mathbb{N}$, il existe $s \in \mathbb{N}$ tel que $\tau^{-j} S_{0} \subset \pi_{1}^{-s} S_{0}$ : en effet, comme $N$ est la réunion des $\pi_{1}^{-s} S_{0}$, la suite des $\tau^{-j} S_{0} \cap \pi_{1}^{-s} S_{0}$, pour $s \in \mathbb{N}$, est une suite croissante de sous- $W$-modules de $\tau^{-j} S_{0}$ dont la réunion est $\tau^{-j} S_{0}$. Comme les éléments de cette suite contiennent $S_{0}$ et comme $\tau^{-j} S_{0} / S_{0}$ est un- $W$-module de longueur finie, il existe $s \in \mathbb{N}$ tel que $\pi_{1}^{-s} S_{0} \cap \tau^{-j} S_{0}=\tau^{-j} S_{0}$ et $\tau^{-j} S_{0} \subset \pi_{1}^{-s} S_{0}$.

Si $T$ est alors un $\tau$-réseau de $N$ et si $S_{0}$ est comme plus haut, il existe des entiers $i, j \in \mathbb{N}$ tels que $\tau^{i} S_{0} \subset T \subset \tau^{-j} S_{0}$ et il suffit de prendre $T_{1}=\pi_{1}^{r} S_{0}$ et $T_{2}=\pi_{1}^{-s} S_{0}$ où $r$ et $s$ sont comme ci-dessus.

3.3.2. Énoncé du théorème. - Rappelons (prop. 3.2) que Ker $\psi$ est un $W\left(\left(\pi_{1}\right)\right)$-module de longueur finie égale à $(p-1) \ell_{V}$. Soit $\mathcal{R}^{\prime}$ un $\pi_{0}$-réseau de $M$ stable par $\phi$ et $\Gamma_{K}$ (un tel réseau existe d'après la prop. 2.4). Pour tout $x \in M$, on a

$$
x=\phi(\psi(x))+(x-\phi(\psi(x)))
$$

donc, dans la décomposition en somme directe $M=\phi(M) \oplus \operatorname{Ker} \psi$, on voit que

$$
\mathcal{R}^{\prime}=\phi\left(\mathcal{R}^{\prime}\right) \oplus\left(\mathcal{R}^{\prime} \cap \operatorname{Ker} \psi\right)
$$

et que $\mathcal{R}^{\prime} \cap \operatorname{Ker} \psi$ est un $\pi_{1}$-réseau de $\operatorname{Ker} \psi$ stable par $\Gamma_{K}$.

ThÉORÈme 3.8. - Le noyau de $\psi$ a la structure suivante :

1) Soit $e_{K}$ l'indice de ramification absolu de $K$. L'action de $\tau$ est bijective sur $\operatorname{Ker} \psi$, qui est un $W((\tau))$-module de longueur finie, égale à $e_{K} \ell_{V}=e_{K} \operatorname{long}_{\mathcal{O}_{\mathcal{E}(K)}}(M)$.

2) Tout $\pi_{1}$-réseau de $\operatorname{Ker} \psi$ stable par $\Gamma_{K}$ est aussi un $\tau$-réseau.

Remarquons que, comme la topologie de $\operatorname{Ker} \psi$ induite par celle de $M$ est sa topologie de $W\left(\left(\pi_{1}\right)\right)$-module de longueur finie, ce théorème, compte tenu de la proposition précédente, implique que cette topologie coincide avec sa topologie de $W((\tau))$-module de longueur finie.

Nous allons d'abord énoncer un lemme, puis en déduire le théorème.

3.3.3. Le lemme fondamental. - Remarquons que si $F$ est un corps valué complet à corps résiduel de caractéristique $p$, le groupe $U_{F}$ des unités fondamentales de $F$ est, de façon naturelle, un $\mathbb{Z}_{p}$-module.

Lemme 3.9. - Supposons l'extension $K_{\infty} / K$ totalement ramifiée, soient $\pi$ une uniformisante de $E_{K}, \omega=\gamma(\pi) / \pi$ et $\alpha \in \mathbb{Z}_{p}$. Rappelons que $\omega$ appartient à $U_{E_{K}}$ (cf. lemme 1.1).

TOME $126-1998-\mathrm{N}^{\circ} 4$ 
1) Il existe sur $E_{K}$ une et une seule action $k[[\tau]]$-linéaire continue

$$
(\lambda, x) \longmapsto \lambda * x \quad \text { pour } \quad \lambda \in k[[\tau]], x \in E_{K},
$$

telle que, pour tout $x \in E_{K}, \lambda * x=\lambda x$ si $\lambda \in k$ et $\gamma * x=\omega^{\alpha} \gamma(x)$.

On suppose que $p$ divise $\alpha$.

2) Alors $\sigma\left(E_{K}\right)$ est un sous- $k[[\tau]]$-module de $E_{K}$ et $\tau$ est inversible sur $E_{K} / \sigma\left(E_{K}\right)$, qui devient ainsi un $k((\tau))$-espace vectoriel de dimension finie égale à $e_{K}$.

3) Sur ce quotient, l'image $S_{\alpha}$ de $\mathcal{O}_{E_{K}}$ est aussi bien un $\tau$-réseau qu'un $\pi_{1}$-réseau.

La démonstration de ce lemme constitue le cour technique de cet article et est l'objet du $\S 5$. Dans la suite, on écrit $E_{K, \alpha}$ pour $E_{K}$ muni de la structure de $k[[\tau]]$-module ainsi définie et, si $p$ divise $\alpha$, on note $\sigma\left(E_{K}\right)_{\alpha}$ le sous-module considéré et $\widetilde{E}_{K, \alpha}$ le quotient $E_{K, \alpha} / \sigma\left(E_{K}\right)_{\alpha}$.

3.3.4. Preuve du théorème. - Rappelons que $n$ désigne un entier tel que $p^{n}$ annule $V$ (et donc aussi $M$ ); pour tout anneau $A$, on note $W_{n}(A)$ l'anneau des vecteurs de Witt de longueur $n$ à coefficients dans $A$.

1) Remplacement de $K$ par une extension finie galoisienne non ramifiée. Soit $K^{\prime}$ une extension finie galoisienne non ramifiée de $K$ contenue dans $\bar{K}$; par restriction $G_{K^{\prime}}=\operatorname{Gal}\left(\bar{K} / K^{\prime}\right)$ opère aussi sur $V$. Rappelons que $M$ s'identifie à $D_{K}(V)=\left(\mathcal{O}_{\mathcal{E}^{\mathrm{nr}}} \otimes_{\mathbb{Z}_{p}} V\right)^{G_{K}} ;$ si $M^{\prime}=D_{K^{\prime}}(V)$, alors $M^{\prime}$ s'identifie à $\left(\mathcal{O}_{\mathcal{E}^{\mathrm{nr}}} \otimes_{\mathbb{Z}_{p}} V\right)^{G_{K^{\prime}}}$ et aussi à $\mathcal{O}_{\mathcal{E}}\left(K^{\prime}\right) \otimes_{\mathcal{O}_{\mathcal{E}(K)}} M$.

Avec des notations évidentes, on voit que

tandis que

$$
\mathcal{O}_{\mathcal{E}(K)}=\mathcal{O}_{\mathcal{E}\left(K^{\prime} \cap K_{\infty}\right)}
$$

$$
\mathcal{O}_{\mathcal{E}\left(K^{\prime}\right)}=W\left(k^{\prime}\right) \otimes_{W\left(k^{\prime} \cap k_{\infty}\right)} \mathcal{O}_{\mathcal{E}\left(K^{\prime} \cap K_{\infty}\right)},
$$

ce qui fait que $M^{\prime}=W_{n}\left(k^{\prime}\right) \otimes_{W_{n}\left(k^{\prime} \cap k_{\infty}\right)} M$, donc aussi que

$$
\operatorname{Ker} \psi_{M^{\prime}}=W_{n}\left(k^{\prime}\right) \otimes_{W_{n}\left(k^{\prime} \cap k_{\infty}\right)} \operatorname{Ker} \psi_{M} \text {. }
$$

De plus, $M=\left(M^{\prime}\right)^{\mathrm{Gal}\left(K^{\prime} / K^{\prime} \cap K_{\infty}\right)}$ et

$$
\operatorname{Ker} \psi_{M}=\left(\operatorname{Ker} \psi_{M^{\prime}}\right)^{\operatorname{Gal}\left(K^{\prime} / K^{\prime} \cap K_{\infty}\right)} .
$$

Supposons le théorème vrai pour $M^{\prime}$. Soient

$$
a=\left(\Gamma_{K}: \Gamma_{K^{\prime}}\right), \quad \gamma^{\prime}=\gamma^{a}, \quad \tau^{\prime}=\gamma^{\prime}-1 .
$$

BULLETIN DE LA SOCIÉTÉ MATHÉMATIQUE DE FRANCE 
Alors $\tau^{\prime}$ est bijectif sur $\operatorname{Ker} \psi_{M^{\prime}}$ qui est un $W_{n}\left(k^{\prime}\right)\left(\left(\tau^{\prime}\right)\right)$-module de longueur finie égale à $e_{K^{\prime}} \ell_{V}=e_{K} \ell_{V}$; on voit aussi que $\tau^{\prime}$, et a fortiori $\tau$, est bijectif sur Ker $\psi_{M}$ et que

$$
\operatorname{Ker} \psi_{M^{\prime}}=W_{n}\left(k^{\prime}\right)\left(\left(\tau^{\prime}\right)\right) \otimes_{W_{N}\left(k^{\prime} \cap k_{\infty}\right)\left(\left(\tau^{\prime}\right)\right)} \operatorname{Ker} \psi_{M}
$$

en particulier, $\operatorname{Ker} \psi_{M}$ est un $W_{n}\left(k^{\prime} \cap k_{\infty}\right)\left(\left(\tau^{\prime}\right)\right)$-module de longueur finie égale à $e_{K} \ell_{V}$.

Par ailleurs, $W_{n}(k)((\tau))$ est libre de rang $a$ sur $W_{n}(k)\left(\left(\tau^{\prime}\right)\right)$, donc

$$
\operatorname{long}_{W_{n}(k)\left(\left(\tau^{\prime}\right)\right)}\left(\operatorname{Ker} \psi_{M}\right)=a \operatorname{long}_{W_{n}(k)((\tau))}\left(\operatorname{Ker} \psi_{M}\right) \text {. }
$$

On a aussi

$$
a=\left[K^{\prime} \cap K_{\infty}: K\right]=\left[k^{\prime} \cap k_{\infty}: k\right]
$$

et $W_{n}\left(k^{\prime} \cap k_{\infty}\right)\left(\left(\tau^{\prime}\right)\right)$ est libre de rang $a$ sur $W_{n}(k)\left(\left(\tau^{\prime}\right)\right)$, donc

$$
\operatorname{long}_{W_{n}(k)\left(\left(\tau^{\prime}\right)\right)}\left(\operatorname{Ker} \psi_{M}\right)=a \operatorname{long}_{W_{n}\left(k^{\prime} \cap k_{\infty}\right)\left(\left(\tau^{\prime}\right)\right)}\left(\operatorname{Ker} \psi_{M}\right),
$$

ce qui fait que

$$
\operatorname{long}_{W_{n}(k)((\tau))}\left(\operatorname{Ker} \psi_{M}\right)=\operatorname{long}_{W_{n}\left(k^{\prime} \cap k \infty\right)\left(\left(\tau^{\prime}\right)\right)}\left(\operatorname{Ker} \psi_{M}\right)=e_{K} \ell_{V},
$$

d'où l'assertion 1) du théorème pour $M$.

Enfin, soient $S$ un $\pi_{1}$-réseau stable par $\Gamma_{K}$ du $W(k)\left(\left(\pi_{1}\right)\right)$-module Ker $\psi_{M}$ et soit $S_{1}$ le sous- $W\left(k^{\prime} \cap k_{\infty}\right)$-module de Ker $\psi_{M}$ engendré par $S$. Alors $S_{1}$ est encore un $\pi_{1}$-réseau de $\operatorname{Ker} \psi_{M}$ stable par $\Gamma_{K}$ et, d'après la proposition 3.7, il suffit de vérifier que $S_{1}$ est bien un $\tau$-réseau. Mais

$$
S^{\prime}=W\left(k^{\prime}\right) \otimes_{W\left(k^{\prime} \cap k_{\infty}\right)} S_{1}
$$

est un $\pi_{1}$-réseau de $\operatorname{Ker} \psi_{M^{\prime}}$ (vu comme $W\left(k^{\prime}\right)\left(\left(\pi_{1}\right)\right)$-module de type fini) stable par $\Gamma_{K}$, donc a fortiori par $\Gamma_{K^{\prime}}$.

Si l'on admet le théorème pour $M^{\prime}$, il en résulte que $S^{\prime}$ est un $\tau^{\prime}$-réseau

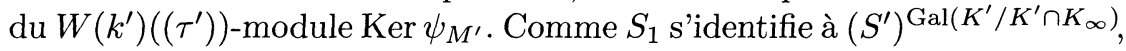
on en déduit que $S_{1}$ est un $\tau^{\prime}$-réseau du $W\left(k^{\prime} \cap k_{\infty}\right)\left(\left(\tau^{\prime}\right)\right)$-module Ker $\psi_{M}$. Puisque $S_{1}$ est stable par $\tau$, c'est alors un $\tau$-réseau de $\operatorname{Ker} \psi_{M}$, vu comme $W(k)((\tau))$-module.

On peut donc, pour prouver le théorème remplacer $K$ par $K^{\prime}$.

2) Réduction au cas où $M$ est simple. On s'y ramène par récurrence sur la longueur de $M$. En effet, soit

$$
0 \rightarrow M^{\prime} \longrightarrow M \longrightarrow M^{\prime \prime} \rightarrow 0
$$

TOME $126-1998-\mathrm{N}^{\circ} 4$ 
une suite exacte de $\Phi-\Gamma_{K}$-modules étales sur $\mathcal{O}_{\mathcal{E}(K)}$. Comme l'action de $\psi$ est surjective sur $M^{\prime}$, elle induit une suite exacte

$$
0 \rightarrow \operatorname{Ker} \psi_{M^{\prime}} \longrightarrow \operatorname{Ker} \psi_{M} \longrightarrow \operatorname{Ker} \psi_{M^{\prime \prime}} \rightarrow 0 .
$$

La bijectivité de $\tau$ sur $\operatorname{Ker} \psi_{M}$ se déduit de la bijectivité de $\tau$ sur Ker $\psi_{M^{\prime}}$ et $\operatorname{Ker} \psi_{M}$ ", de même que l'assertion sur la longueur de $\operatorname{Ker} \psi_{M}$ résulte des assertions analogues pour $\operatorname{Ker} \psi_{M^{\prime}}$ et $\operatorname{Ker} \psi_{M}$ ".

Si $S$ est un $\pi_{1}$-réseau de $\operatorname{Ker} \psi_{M}$ stable par $\Gamma_{K}$, son image $S^{\prime \prime}$ dans Ker $\psi_{M^{\prime \prime}}$ est un $\pi_{1}$-réseau de $\operatorname{Ker} \psi_{M^{\prime \prime}}$ stable par $\Gamma_{K}$, tandis que le noyau $S^{\prime}$ de la projection de $S$ sur $S$ " est un $\pi_{1}$-réseau de Ker $\psi_{M^{\prime}}$ stable par $\Gamma_{K}$. Le fait que $S$ soit un $\tau$-réseau de $\operatorname{Ker} \psi_{M}$ résulte alors de ce que $S^{\prime}$ est un $\tau$-réseau de $\operatorname{Ker} \psi_{M^{\prime}}$ et $S^{\prime \prime}$ un $\tau$-réseau de $\operatorname{Ker} \psi_{M^{\prime \prime}}$.

3) Fin de la preuve. Soient $G_{V}$ le noyau de l'action de $G_{K}$ sur $V$, $L=\bar{K}^{G_{V}}$ et $J=\operatorname{Gal}(L / K)$. Grâce à 1$)$ on peut supposer les extensions $K_{\infty} / K$ et $L / K$ totalement ramifiées. Le fait que $L / K$ le soit implique ( $c f$. [Se1, cor. 4, p. 75$]$ ) que $J$ est le produit semi-direct d'un $p$-groupe invariant $P$ par un groupe cyclique $J^{\prime}$ dont l'ordre $m$ est premier à $p$. Grâce à 2), on peut supposer que $V$ est simple; ceci entraîne que $V$ est tuée par $p$ et aussi que $P$ est trivial (car $V^{P}$ est un sous- $\mathbb{F}_{p}[J]$-module de $V$ non trivial : cf. [Se1, th. 2, p. 146]), donc que $J=J^{\prime}$.

Soit $E_{L}=\left(E^{\text {sep }}\right)^{G_{V} \cap \operatorname{Gal}\left(\bar{K} / K_{\infty}\right)}$. Le groupe de Galois (respectivement le groupe d'inertie) de l'extension $E_{L} / E_{K}$ s'identifie à l'image du groupe $\operatorname{Gal}\left(\bar{K} / K_{\infty}\right)$ (respectivement du sous-groupe d'inertie de $\operatorname{Gal}\left(\bar{K} / K_{\infty}\right)$ ) dans $J$. Comme $J$ est d'ordre premier à $p$, cette image est $J$ et $E_{L} / E_{K}$ est une extension totalement ramifiée, cyclique d'ordre $m$. Si $q$ est la plus petite puissance de $p$ telle que $m$ divise $q-1$, ceci entraîne ( $c f$. [Se1, cor. 1 , p. 75]) que le corps résiduel $k$ de $E_{K}$ contient un corps à $q$ éléments, que nous notons $F_{0}$, qu'il existe une uniformisante $\pi_{L}$ de $E_{L}$ telle que $\pi=\pi_{L}^{m}$ est une uniformisante de $E_{K}$ et que le caractère

$$
\eta_{0}: J \longrightarrow F_{0}^{*}
$$

défini par $\eta_{0}(g)=g\left(\pi_{L}\right) / \pi_{L}$, engendre le groupe dual de $J$.

Par ailleurs, si $V^{*}=\operatorname{Hom}_{\mathbb{F}_{p}}\left(V, \mathbb{F}_{p}\right)$ désigne la représentation duale, on voit que $V^{*}$ est de dimension 1 sur le corps

$$
F=\operatorname{End}_{\mathbb{F}_{p}\left[\operatorname{Gal}\left(\bar{K} / K_{\infty}\right)\right]}(V)=\operatorname{End}_{\mathbb{F}_{p}[J]}(V),
$$

qui est aussi un corps à $q$ éléments. On choisit un générateur de $V^{*}$ sur $F$, ce qui nous permet d'identifier $V^{*}$ à $F$ muni de l'action de $J$ donnée par un caractère $\eta: J \rightarrow F^{*}:$ pour $g \in J$ et $v^{*} \in V^{*}$, on a donc $g\left(v^{*}\right)=\eta(g) v^{*}$. 
La projection de $M$ sur $M / \phi(M)$ induit un isomorphisme, compatible avec toutes les structures, de $\operatorname{Ker} \psi$ sur $M / \phi(M)$, ce qui nous permet, pour prouver le théorème, de remplacer $\operatorname{Ker} \psi \operatorname{par} M / \phi(M)$.

Si $G=\operatorname{Gal}\left(\bar{K} / K_{\infty}\right)$, on a

$$
\begin{aligned}
M & =\left(\mathcal{O}_{\mathcal{E}^{\mathrm{nr}}} \otimes_{\mathbb{Z}_{p}} V\right)^{G}=\left(E^{\mathrm{sep}} \otimes_{\mathbb{F}_{p}} V\right)^{G} \\
& =\operatorname{Hom}_{\mathbb{F}_{p}[G]}\left(V^{*}, E^{\mathrm{sep}}\right)=\operatorname{Hom}_{\mathbb{F}_{p}[J]}\left(V^{*}, E_{L}\right)
\end{aligned}
$$

et c'est un $E_{K^{-}}$-espace vectoriel de dimension $\ell_{V}=\operatorname{dim}_{\mathbb{F}_{p}}(V)=\left[F: \mathbb{F}_{p}\right]$.

L'ensemble $T$ des $\mathbb{F}_{p}$-plongements de $F$ dans $F_{0}$ a $\operatorname{dim}_{\mathbb{F}_{p}}\left(V^{*}\right)=\ell_{V}$ éléments. Pour chaque $t \in T$, notons $a_{t}$ l'unique entier vérifiant $0 \leq a_{t}<m$ tel que $t \circ \eta=\eta_{0}^{a_{t}}$. Notons

$$
e_{t}: V^{*} \longrightarrow E_{L}
$$

l'application définie par

$$
e_{t}\left(v^{*}\right)=t\left(v^{*}\right) \pi_{L}^{a_{t}}
$$

Pour tout $g \in J$ et tout $v^{*} \in V^{*}$, on a alors :

$$
e_{t}\left(g\left(v^{*}\right)\right)=t\left(\eta(g) v^{*}\right) \pi_{L}^{a_{t}}=\eta_{0}^{a_{t}}(g) t\left(v^{*}\right) \pi_{L}^{a_{t}}=g\left(t\left(v^{*}\right)\right) \pi_{L}^{a_{t}}=g\left(e_{t}\left(v^{*}\right)\right) .
$$

Les $e_{t}$ sont donc dans $M$ et ils sont linéairement indépendants sur $E_{K}$; de ce fait, ils forment une base de $M$ sur $E_{K}$. Si $e_{t}^{\prime}=\phi\left(e_{t}\right)=\sigma \circ e_{t}$, il en est de même des $e_{t}^{\prime}$. Les applications

$$
\theta:\left(E_{K}\right)^{T} \longrightarrow M, \quad \theta^{\prime}:\left(E_{K}\right)^{T} \longrightarrow M
$$

définies par

$$
\theta\left(\left(x_{t}\right)_{t \in T}\right)=\sum_{t \in T} x_{t} e_{t}, \quad \theta\left(\left(y_{t}\right)_{t \in T}\right)=\sum_{t \in T} y_{t} e_{t}^{\prime}
$$

sont donc des isomorphismes de $E_{K}$-espaces vectoriels.

L'action de $\Gamma_{K}$ sur $E_{K}$ s'étend de manière unique à $E_{L}:$ si $\gamma(\pi)=\omega \pi$, on a $\gamma\left(\pi_{L}\right)=\omega^{1 / m} \pi_{L}$. Pour tout $t \in T, e_{t}^{\prime}(v)=t\left(v^{p}\right) \pi_{L}^{p a_{t}}$ et on en déduit que, si $\alpha_{t}=p a_{t} / m$, on a $\gamma\left(e_{t}^{\prime}\right)=\omega^{\alpha_{t}} e_{t}^{\prime}$. Par conséquent, l'application $\theta^{\prime}$ peut aussi être considérée comme un isomorphisme de $k[[\tau]]$-modules

$$
\theta^{\prime}: \bigoplus_{t \in T} E_{K, \alpha_{t}} \longrightarrow M
$$

Si l'on écrit un élément $x \in M$ sous la forme $x=\sum_{t \in T} x_{t} e_{t}$, avec les $x_{t} \in E_{K}$, on voit que $\phi(x)=\sum_{t \in T} x_{t}^{p} e_{t}^{\prime}$. On obtient ainsi, par passage

TOME $126-1998-\mathrm{N}^{\circ} 4$ 
aux quotients à partir de l'inverse de $\theta^{\prime}$ une bijection

$$
\nu: M / \phi(M) \longrightarrow \bigoplus_{t \in T} \widetilde{E}_{K, \alpha_{t}},
$$

qui est aussi bien un isomorphisme de $\sigma\left(E_{K}\right)$-espaces vectoriels que de $k[[\tau]]$-modules.

La première partie du théorème résulte alors de la deuxième assertion du lemme 3.9. D'après la proposition 3.7 , il suffit de vérifier la deuxième partie pour un $\pi_{1}$-réseau $S$ de $M / \phi(M)$ stable par $\Gamma_{K}$ particulier. Si, avec les notations du lemme 3.9, on prend pour $S$ l'image inverse par $\nu$ de $\bigoplus_{t \in T} S_{\alpha_{t}}$, la troisième assertion du lemme permet de conclure.

\subsection{Le cœur de $M$.}

DÉfinition 3.10. - Si $N$ est un $W((X))$-module de longueur finie, on appelle $X$-pseudo-réseau de $N$ tout sous-groupe $\Lambda$ de $N$ stable par $X$ qui contient un $X$-réseau $\Lambda_{1}$ et est contenu dans un $X$-réseau $\Lambda_{2}$ de $N$.

Autrement dit, un $X$-pseudo-réseau est un sous-groupe ouvert stable par $X$ qui est contenu dans un $X$-réseau.

Si $k$ est fini, $N$ est un $\mathbb{Z}_{p}((X))$-module de longueur finie égale à $\left[k: \mathbb{F}_{p}\right] \operatorname{long}_{W((X))}(N)$. Un $X$-pseudo-réseau n'est alors rien d'autre qu'un $X$-réseau de $N$, considéré comme $\mathbb{Z}_{p}((X))$-module.

On appelle cœur de $M$ le sous $-\mathbb{Z}_{p}[[\tau]]$-module de $M$ :

$$
c(M)=(\phi-1) M \cap \operatorname{Ker} \psi .
$$

Si $x \in M$, alors $(\phi-1)(x) \in \operatorname{Ker} \psi$ si et seulement si $\psi(x)=x$ de sorte que $c(M)=(\phi-1)\left(M_{\psi=1}\right)$.

Proposition 3.10. - Le cœur de $M$ est un $\tau$-pseudo-réseau de Ker $\psi$.

Preuve. - On sait (prop. 2.4) que $M$ contient un $\pi_{0}$-réseau $\mathcal{R}$ sur lequel $\rho=\phi-1$ est bijective; donc le groupe $(\phi-1) M$ contient le $\pi_{0}$-réseau $\mathcal{R}$ et est ouvert dans $M$. Par conséquent $(\phi-1) M \cap \operatorname{Ker} \psi=c(M)$ est ouvert dans $\operatorname{Ker} \psi$.

Soit $\mathcal{N}$ comme dans la proposition 3.3. On a $M_{\psi=1} \subset \mathcal{N}$ et de ce fait :

$$
c(M)=(\phi-1)\left(M_{\psi=1}\right) \subset(\phi-1)(\mathcal{N}) \cap \operatorname{Ker} \psi \subset(\mathcal{N}+\phi(\mathcal{N})) \cap \operatorname{Ker} \psi
$$

Mais $\mathcal{N}$ est un sous- $W\left[\left[\pi_{0}\right]\right]$-module de type fini de $M$, donc $\phi(\mathcal{N})$ est un sous- $W\left[\left[\pi_{1}\right]\right]$-module de type fini de $M$; comme $W\left[\left[\pi_{0}\right]\right]$ est fini sur 
$W\left[\left[\pi_{1}\right]\right]$, on voit que $\mathcal{N}$ est aussi un sous- $W\left[\left[\pi_{1}\right]\right]-$ module de type fini de $M$; il en est donc de même de $\mathcal{N}+\phi(\mathcal{N})$. Finalement, $(\mathcal{N}+\phi(\mathcal{N})) \cap \operatorname{Ker} \psi$ est contenu dans un sous- $W\left[\left[\pi_{1}\right]\right]$-module de type fini de Ker $\psi$, donc dans un $\pi_{1}$-réseau de $\operatorname{Ker} \psi$. L'assertion résulte alors de ce que, compte tenu de la proposition 3.7 , le théorème 3.8 implique que tout $\pi_{1}$-réseau de $\operatorname{Ker} \psi$ est contenu dans un $\tau$-réseau.

Corollaire 3.11. - Si $\left[K: \mathbb{Q}_{p}\right]$ est fini, $c(M) / \tau(c(M))$ est un groupe fini d'ordre $p^{\left[K: \mathbb{Q}_{p}\right] \ell_{V}}$.

Comme $\operatorname{Ker} \psi$ est un $\mathbb{Z}_{p}((\tau))$-module de longueur

$$
\left[k: \mathbb{F}_{p}\right] \operatorname{long}_{W((\tau))}(\operatorname{Ker} \psi)=\left[k: \mathbb{F}_{p}\right] e_{K} \ell_{V}=\left[K: \mathbb{Q}_{p}\right] \ell_{V},
$$

ce corollaire résulte du lemme suivant :

Lemme 3.12. - Si A est un $\mathbb{Z}_{p}((X))$-module de type fini tué par une puissance de $p$ et $B$ un $X$-réseau de $A$, alors $B / X B$ est un groupe fini d'ordre $p^{\ell}$, où $\ell$ est la longueur de $A$ sur $\mathbb{Z}_{p}((X))$.

Preuve. - Remarquons que $p A \cap B$ et $B /(p A \cap B)$ sont des $X$-réseaux de $p A$ et $A / p A$ respectivement. De plus, comme $A$ est sans $X$-torsion, le lemme du serpent donne la suite exacte :

$$
0 \rightarrow \frac{p A \cap B}{X(p A \cap B)} \longrightarrow \frac{B}{X B} \longrightarrow \frac{B /(p A \cap B)}{X(B /(p A \cap B))} \rightarrow 0 .
$$

Par récurrence sur la plus petite puissance de $p$ qui annule $A$, on peut donc supposer que $p A=0$. Dans ce cas, $A$ est un $\mathbb{F}_{p}((X))$-espace vectoriel de dimension finie $\ell$ et $B$ est un sous- $\mathbb{F}_{p}[[X]]$-module libre de rang $\ell$. Donc $B / X B$ est un $\mathbb{F}_{p}$-espace vectoriel de dimension $\ell$, i.e. un groupe fini d'ordre $p^{\ell}$.

\section{Calcul de la cohomologie galoisienne}

Rappelons que $n$ est un entier $\geq 1, M$ un $\Phi$ - $\Gamma_{K}$-module étale sur $\mathcal{O}_{\mathcal{E}(K)}$ tué par $p^{n}, V=V_{K}(M)$ et $\ell_{V}=\operatorname{long}_{\mathbb{Z}_{p}}(V)$.

Dans ce paragraphe, on adopte la convention suivante : dans tout complexe considéré, le premier terme écrit est placé en degré -1.

\subsection{Un complexe filtré quasi-isomorphe à $\mathcal{C}_{\phi, \gamma}(M)$.}

Rappelons que la cohomologie galoisienne de $V$ est la cohomologie du complexe $\mathcal{C}_{\phi, \gamma}(M)$. On va commencer par montrer que cette cohomologie peut aussi se calculer en remplaçant $\phi$ par $\psi$ dans les flèches de ce complexe. De façon précise :

$$
\text { TOME } 126-1998-\mathrm{N}^{\circ} 4
$$


Proposition 4.1. - Soit $\mathcal{C}_{\psi, \gamma}(M)$ le complexe

$$
\begin{gathered}
0 \longrightarrow M \longrightarrow M \oplus M \longrightarrow M \longrightarrow 0 \longrightarrow 0 \rightarrow \cdots, \\
x \longmapsto((\psi-1)(x), \tau(x)), \\
(y, z) \longmapsto \tau(y)-(\psi-1)(z) .
\end{gathered}
$$

Soit $f: M \oplus M \rightarrow M \oplus M$ définie par $(y, z) \mapsto(-\psi(y), z)$. Alors, le morphisme de complexes

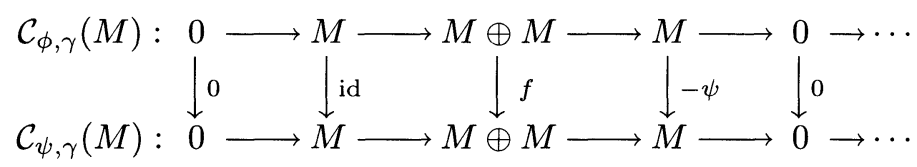

est un quasi-isomorphisme.

Preuve. - Comme $\psi$ est surjective, on voit que $\mathcal{C}_{\psi, \gamma}(M)$ est le quotient, via le morphisme de complexes précédent, de $\mathcal{C}_{\phi, \gamma}(M)$ par le souscomplexe :

$$
0 \rightarrow 0 \longrightarrow \operatorname{Ker} \psi \oplus 0 \longrightarrow \operatorname{Ker} \psi \rightarrow 0 \rightarrow \cdots .
$$

et la bijectivité de $\tau$ sur $\operatorname{Ker} \psi$ signifie que ce sous-complexe est acyclique.

\subsection{La filtration canonique de $\mathcal{C}_{\boldsymbol{\psi}, \boldsymbol{\gamma}}(M)$.}

Pour étudier la cohomologie du complexe $\mathcal{C}(M)=\mathcal{C}_{\psi, \gamma}(M)$, on va le munir d'une filtration en trois crans par des sous-complexes

$$
\mathcal{C}_{\psi, \gamma}(M)=\mathcal{C}^{0}(M) \supset \mathcal{C}^{1}(M) \supset \mathcal{C}^{2}(M) \supset \mathcal{C}^{3}(M)
$$

en posant

$$
\begin{aligned}
& \mathcal{C}^{1}(M): 0 \rightarrow M_{\psi=1} \stackrel{\tau}{\longrightarrow} M_{\psi=1} \longrightarrow 0 \longrightarrow 0 \rightarrow \cdots, \\
& \mathcal{C}^{2}(M): 0 \rightarrow M_{\phi=1} \stackrel{\tau}{\longrightarrow} M_{\phi=1} \longrightarrow 0 \longrightarrow 0 \rightarrow \cdots,
\end{aligned}
$$

et $\mathcal{C}^{3}(M)$ est le complexe nul.

Pour $i \in\{0,1,2\}$, on définit

$$
\operatorname{gr}^{i} \mathcal{C}(M)=\mathcal{C}^{i}(M) / \mathcal{C}^{i+1}(M)
$$

Remarquons que, comme $M_{\phi=1}$ s'identifie à $V^{\mathrm{Gal}\left(\bar{K} / K_{\infty}\right)}$, le complexe $\operatorname{gr}^{2} \mathcal{C}(M)=\mathcal{C}^{2}(M)$ s'identifie au complexe

$$
0 \rightarrow V^{\operatorname{Gal}\left(\bar{K} / K_{\infty}\right)} \stackrel{\tau}{\longrightarrow} V^{\operatorname{Gal}\left(\bar{K} / K_{\infty}\right)} \longrightarrow 0 \longrightarrow 0 \rightarrow \cdots .
$$

Le complexe $\operatorname{gr}^{1} \mathcal{C}(M)$ est

$$
0 \rightarrow M_{\psi=1} / M_{\phi=1} \stackrel{\tau}{\longrightarrow} M_{\psi=1} / M_{\phi=1} \longrightarrow 0 \longrightarrow 0 \rightarrow \cdots .
$$

Pour $i=0$, on a la description suivante :

$$
\text { BULLETIN DE LA SOCIÉTÉ MATHÉMATIQUE DE FRANCE }
$$


Lemme 4.2. - Le complexe $\operatorname{gr}^{0} \mathcal{C}(M)$ est quasi-isomorphe à :

$$
0 \rightarrow 0 \longrightarrow \frac{M}{(\psi-1)(M)} \stackrel{\tau}{\longrightarrow} \frac{M}{(\psi-1)(M)} \longrightarrow 0 \rightarrow \cdots
$$

Preuve. - il suffit de montrer que le sous-complexe suivant de $\operatorname{gr}^{0} \mathcal{C}(M):$

$$
0 \rightarrow \frac{M}{M_{\psi=1}} \longrightarrow(\psi-1)(M) \oplus \frac{M}{M_{\psi=1}} \longrightarrow(\psi-1)(M) \rightarrow 0 \rightarrow \cdots
$$

est acyclique, ce qui résulte imédiatement de ce que $\psi-1$ induit une bijection de $M / M_{\psi=1}$ sur $(\psi-1)(M)$.

\subsection{La filtration associée sur la cohomologie.}

L'anneau $\mathbb{Z}_{p}\left[\left[\Gamma_{K}\right]\right]$ s'identifie à l'anneau $\mathbb{Z}_{p}[[\tau]]$ des séries formelles en $\tau$ à coefficients dans $\mathbb{Z}_{p}$. Pour tout $\mathbb{Z}_{p}\left[\left[\Gamma_{K}\right]\right]$-module topologique $N$, on note $\left(H^{i}\left(\Gamma_{K}, N\right)\right)_{i \in \mathbb{Z}}$ les groupes de cohomologie du complexe

$$
0 \rightarrow N \stackrel{\tau}{\longrightarrow} N \longrightarrow 0 \longrightarrow 0 \rightarrow \cdots
$$

Lorsque $N$ est un $\Gamma_{K}$-module discret, on voit facilement que ces groupes s'identifient aux groupes de cohomologie habituels.

On a défini une filtration en trois crans du complexe $\mathcal{C}_{\psi, \gamma}(M)$; la suite exacte longue de cohomologie associée à une suite exacte courte de complexes donne alors une filtration en trois crans des groupes de cohomologie $\mathcal{H}^{i}(M)$ :

ThÉORÈme 4.3. - Pour tout entier naturel $i$, on a :

- $H^{i}\left(\operatorname{gr}^{0} \mathcal{C}(M)\right)=H^{i-1}\left(\Gamma_{K}, M /(\psi-1)(M)\right)$,

- $H^{i}\left(\operatorname{gr}^{1} \mathcal{C}(M)\right)=\{0\}$ sauf si $i=1$ et $H^{1}\left(\operatorname{gr}^{1} \mathcal{C}(M)\right)=c(M) / \tau(c(M))$,

- $H^{i}\left(\operatorname{gr}^{2} \mathcal{C}(M)\right)=H^{i}\left(\Gamma_{K}, M_{\phi=1}\right)=H^{i}\left(\Gamma_{K}, V^{\operatorname{Gal}\left(\bar{K} / K_{\infty}\right)}\right)$

ainsi que la filtration suivante :

$$
\mathcal{H}^{i}(M) \supset H^{i}\left(\Gamma_{K}, M_{\psi=1}\right) \supset H^{i}\left(\Gamma_{K}, M_{\phi=1}\right),
$$

avec

$$
\frac{\mathcal{H}^{i}(M)}{H^{i}\left(\Gamma_{K}, M_{\psi=1}\right)} \simeq H^{i}\left(\operatorname{gr}^{0} \mathcal{C}(M)\right) \quad \text { et } \quad \frac{H^{i}\left(\Gamma_{K}, M_{\psi=1}\right)}{H^{i}\left(\Gamma_{K}, M_{\phi=1}\right)} \simeq H^{i}\left(\operatorname{gr}^{1} \mathcal{C}(M)\right)
$$

Preuve. - Les assertions concernant $\operatorname{gr}^{0} \mathcal{C}(M)$ et $\operatorname{gr}^{2} \mathcal{C}(M)$ sont évidentes. Pour $\operatorname{gr}^{1} \mathcal{C}(M)$, on remarque que $\phi-1$ induit un isomorphisme $\Gamma_{K^{-}}$ équivariant de $M_{\psi=1} / M_{\phi=1} \operatorname{sur}(\phi-1)\left(M_{\psi=1}\right)=(\phi-1) M \cap \operatorname{Ker} \psi=c(M)$. L'injectivité de l'action de $\tau$ sur Ker $\psi$ permet de conclure. Le reste découle alors immédiatement de l'examen de suites exactes longues de cohomologie et de la nullité de certains groupes.

TOME $126-1998-\mathrm{N}^{\circ} 4$ 


\subsection{Preuve du théorème $B$.}

Compte-tenu des résultats du $\S 3$, le théorème $\mathrm{B}$ se déduit immédiatement du théorème 4.3. En effet, $V^{\mathrm{Gal}\left(\bar{K} / K_{\infty}\right)}$ est un groupe fini et les $H^{i}\left(\operatorname{gr}^{2} \mathcal{C}(M)\right)$ sont triviaux sauf peut-être $H^{0}$ et $H^{1}$ qui sont finis du même ordre. Si l'extension $K / \mathbb{Q}_{p}$ est finie, comme $M /(\psi-1)(M)$ est un groupe fini (proposition 3.6), les $H^{i}\left(\operatorname{gr}^{0} \mathcal{C}(M)\right)$ sont triviaux sauf peut-être $H^{1}$ et $H^{2}$ qui sont finis du même ordre; enfin, les $H^{i}\left(\operatorname{gr}^{1} \mathcal{C}(M)\right)$ sont triviaux sauf peut-être $H^{1}$ qui est un groupe fini d'ordre $p^{\left[K: \mathbb{Q}_{p}\right] \ell_{V}}$ (cor. 3.11).

\section{Preuve du lemme fondamental}

L'objet essentiel de ce paragraphe est d'établir le lemme 3.9. On va en fait démontrer un résultat un peu plus général, à savoir le même énoncé en remplaçant $K_{\infty}$ par une $\mathbb{Z}_{p}$-extension totalement ramifiée arbitraire de $K$. De façon précise :

ThÉORÈme 5.1. - Soit $K_{\infty}$ une $\mathbb{Z}_{p}$-extension totalement ramifiée de $K$. Soient $\Gamma=\operatorname{Gal}\left(K_{\infty} / K\right), \gamma$ un générateur topologique de $\Gamma$ et $\tau=\gamma-1$. Soient $E$ le corps des normes de l'extension $K_{\infty} / K, \pi$ une uniformisante de $E, \omega=\gamma(\pi) / \pi$ et $\alpha \in \mathbb{Z}_{p}$. L'élément $\omega$ est une unité fondamentale de $E$.

1) Il existe sur $E$ une et une seule action $k[[\tau]]$-linéaire continue

$$
(\lambda, x) \longmapsto \lambda * x \quad \text { pour } \quad \lambda \in k[[\tau]], x \in E,
$$

telle que, pour tout $x \in E, \lambda * x=\lambda x$ si $\lambda \in k$ et $\gamma * x=\omega^{\alpha} \gamma(x)$.

On suppose que $p$ divise $\alpha$.

2) Alors $\sigma(E)$ est un sous- $k[[\tau]]-$ module de $E$ et $\tau$ est inversible sur $E / \sigma(E)$ qui devient ainsi un $k((\tau))$-espace vectoriel de dimension finie égale à $e_{K}$.

3) Sur ce quotient, l'image $S_{\alpha}$ de l'anneau des entiers $\mathcal{O}_{E}$ de $E$ est aussi bien un $\tau$-réseau qu'un $\pi_{1}$-réseau.

L'assertion 1) est évidente. Dans toute la suite on suppose que le nombre $p$-adique $\alpha$ est divisible par $p$. Le fait que $\sigma(E)$ est un sous- $k[[\tau]]-$ module de $E$ est aussi évident. Pour prouver le reste, nous allons avoir besoin d'un résultat sur les nombres de ramification de l'extension $K_{\infty} / K$.

\subsection{Les nombres de ramification de $\gamma$.}

Remarquons d'abord que le corps résiduel de $E$ est $k$ sur lequel $\Gamma$ opère trivialement. En particulier, pour tout $g \in \Gamma,(g(\pi)-\pi) / \pi$ appartient à l'idéal maximal de $\mathcal{O}_{E}$. Notons $v$ la valuation sur $E$ normalisée par 
$v(\pi)=1$. On définit alors les nombres de ramification de $\gamma$, en posant, pour tout $n \in \mathbb{N}$,

$$
i_{n}(\gamma)=v\left(\frac{\gamma^{p^{n}}(\pi)}{\pi}-1\right)
$$

Comme $\Gamma$ opère fidèlement sur $E$, ce sont des entiers $\geq 1$. On vérifie que la suite des $i_{n}(\gamma)$ est strictement croissante et que, si $v_{p}$ est la valuation $p$-adique,

$$
\forall m \in \mathbb{Z}_{p}, \quad v\left(\frac{\gamma^{m}(\pi)}{\pi}-1\right)=i_{v_{p}(m)}(\gamma)
$$

Proposition 5.2. - Les nombres de ramification de $\gamma$ vérifient:

1) pour tout $n \in \mathbb{N}, i_{n+1}(\gamma) \equiv i_{n}(\gamma) \quad\left(\bmod p^{n+1}\right)$;

2) pour tout $n$ suffisamment grand, $i_{n+1}(\gamma)-i_{n}(\gamma)=e_{K} p^{n+1}$, où $e_{K}$ est l'indice de ramification absolu de $K$.

Preuve. - Posons $u_{0}=i_{0}(\gamma)$, et pour tout entier $n \geq 1$,

$$
u_{n}=u_{n-1}+\left(i_{n}(\gamma)-i_{n-1}(\gamma)\right) / p^{n}
$$

La première assertion signifie que les $u_{n}$ sont des entiers et la seconde que $u_{n}-u_{n-1}$ est constant pour $n$ assez grand. Mais, d'après [Win, cor. 3.3.4], les $u_{n}$ ne sont autres que les nombres de ramification en numérotation supérieure de la $\mathbb{Z}_{p}$-extension $K_{\infty} / K$. Alors 1) n'est autre que le théorème de Hasse-Arf ( $c f$. [Se1, p. 84]) et 2) est aussi un résultat classique ( $c f$. [Wym, $\S 5]$ ) qui peut s'obtenir soit par la théorie du corps de classes, soit par des calculs un peu pénibles mais élémentaires.

\section{REMARQUes.}

a) En fait, la preuve de ces résultats sur les nombres de ramification de l'extension $K_{\infty} / K$ est beaucoup plus simple dans le cas particulier où nous en avons besoin, celui où $K_{\infty}$ est la $\mathbb{Z}_{p}$-extension cyclotomique de $K$ (supposée totalement ramifiée). Lorsque $K$ est le corps obtenu en adjoignant les racines $2 p$-ièmes de 1 au corps des fractions de $W$, un calcul facile montre que, pour tout $n \in \mathbb{N}, i_{n}=p^{n+1}-1$ si $p \neq 2$ (resp. $p^{n+2}-1$ si $p=2$ ) ( $c f$. [Se1, prop. 17, p. 85]); le cas général s'en déduit en utilisant le théorème de Herbrand ( $c f$. [Se1, p. 82]).

b) L'assertion 1) est aussi un cas particulier d'un théorème de Sen [Sen, th. 1]) valable pour tout «automorphisme sauvagement ramifié 》de n'importe quel corps complet pour une valuation discrète à corps résiduel parfait.

TOME $126-1998-\mathrm{N}^{\circ} 4$ 
Corollaire 5.3. - On a

$$
\lim _{n \rightarrow+\infty} \frac{i_{n}(\gamma)}{p^{n}}=\frac{p e_{K}}{p-1}
$$

et pour $n$ suffisamment grand:

$$
i_{n+1}(\gamma)<(2 p-1) i_{n}(\gamma)
$$

Preuve. - On déduit de 2) qu'il existe un entier $N$ tel que

$$
\forall n \geq N, \quad i_{n}(\gamma)=i_{N}(\gamma)+p^{N+1} e_{K} \frac{p^{n-N}-1}{p-1}
$$

et la limite est évidente. On a donc, pour $n$ suffisamment grand :

$$
\frac{i_{n}(\gamma)}{p^{n}}>\frac{p e_{K}}{2(p-1)},
$$

ou encore

$$
i_{n}(\gamma)+e_{K} p^{n+1}<(2 p-1) i_{n}(\gamma),
$$

c'est-à-dire $i_{n+1}(\gamma)<(2 p-1) i_{n}(\gamma)$ si en outre $n \geq N$.

\subsection{Le calcul de $\boldsymbol{v}_{\mathbf{0}}\left(\boldsymbol{\tau}^{\boldsymbol{s}} * \overline{\boldsymbol{x}}\right)$ pour $\boldsymbol{s} \in \mathbb{N}, \overline{\boldsymbol{x}} \in \boldsymbol{E} / \sigma(\boldsymbol{E})$.}

On note $v_{0}: E / \sigma(E) \rightarrow \mathbb{Z} \cup\{\infty\}$ l'application définie par :

$$
\forall \bar{x} \in E / \sigma(E), \quad v_{0}(\bar{x})=\sup \{v(x), x \in \bar{x}\} .
$$

Cette borne supérieure est atteinte. Si $\bar{x}$ est non nul, $v_{0}(\bar{x})$ est un entier premier à $p$, sinon $v_{0}(\bar{x})$ est infini.

Lemme 5.4. - Pour tout entier $n$ premier à $p$, choisissons un élément $\bar{x}_{n}$ de $E / \sigma(E)$ tel que $v_{0}\left(\bar{x}_{n}\right)=n$. Alors tout élément $\bar{x}$ de $E / \sigma(E)$ s'écrit de manière unique sous la forme:

$$
\bar{x}=\sum_{\substack{n \gg-\infty \\(n, p)=1}} \lambda_{n} \bar{x}_{n}, \quad \text { avec } \lambda_{n} \in k \text { pour tout } n
$$

et $v_{0}(\bar{x})$ est le plus petit entier $n$ tel que $\lambda_{n} \neq 0$.

Preuve. - Pour tout $n$ premier à $p$, choisissons un relèvement $x_{n}$ de $\bar{x}_{n}$ dans $E$ tel que $v\left(x_{n}\right)=n$; pour tout $n$ divisible par $p$, posons $x_{n}=\pi^{n}$. Alors tout $x \in E$ s'écrit sous la forme $\sum_{n \geq v(x)} \lambda_{n} x_{n}$ où les $\lambda_{n}$ sont des éléments de $k$. Par réduction modulo $\sigma(E)$, on obtient l'écriture voulue. Le reste du lemme est évident. 
Lemme 5.5. - Pour tout $s \in \mathbb{N}$, posons $\omega_{s}=\prod_{0 \leq i<s} \gamma^{i}(\omega)$.

1) On a $\gamma^{s}(\pi) / \pi=\omega_{s}$ et, pour tout $x \in E, \gamma^{s} * x=\omega_{s}^{\alpha} \gamma^{s}(x)$.

2) Si s est une puissance de $p$, pour tout $x \in E, \tau^{s} * x=\left(\omega_{s}^{\alpha} \gamma^{s}-1\right)(x)$.

Preuve. - L'assertion 1) est immédiate par récurrence sur $s$ et 2) résulte alors de ce que, si $s$ est une puissance de $p$, on a $\tau^{s}=(\gamma-1)^{s}=\left(\gamma^{s}-1\right)$ dans $k[[\tau]]$.

Lemme 5.6. - Pour tout $n \in \mathbb{Z}_{p}$ et tout $s \in \mathbb{N}$, on $a$ :

$$
v\left(\omega_{s}^{n}-1\right)=p^{v_{p}(n)} i_{v_{p}(s)}(\gamma)
$$

Preuve. - Tout $n \in \mathbb{Z}_{p}$ s'écrit sous la forme $p^{v_{p}(n)} m$ avec $m$ unité $p$-adique. On a alors :

$$
v\left(\omega_{s}^{n}-1\right)=v\left(\left(\omega_{s}^{m}-1\right)^{p^{v_{p}(n)}}\right)=p^{v_{p}(n)} v\left(\omega_{s}^{m}-1\right) .
$$

Comme $m$ est premier à $p$, on a $v\left(\omega_{s}^{m}-1\right)=v\left(\omega_{s}-1\right)=i_{v_{p}(s)}(\gamma)$

Lemme 5.7. - Pour tout $r \in \mathbb{N}$ et tout $x \in E$, on $a$ :

1) $v\left(\tau^{p^{r}} * x\right) \geq v(x)+i_{r}(\gamma)$ avec égalité si $(v(x), p)=1$.

2) $v\left(\tau^{p^{r}} * x\right) \geq v(x)+p i_{r}(\gamma)$ si $x \in \sigma(E)$.

Preuve. - D'après le lemme 5.5, pour tout $n \in \mathbb{Z}$, on a $\tau^{p^{r}} * \pi^{n}=$ $\omega_{p^{r}}^{\alpha}\left(\omega_{p^{r}} \pi\right)^{n}-\pi^{n}=\left(\omega_{p^{r}}^{\alpha+n}-1\right) \pi^{n}$ et, compte-tenu du lemme 5.4, 1) et 2) sont des conséquences immédiates du lemme 5.6.

Lemme 5.8. - Soient $x \in E$ et $\bar{x}$ sa classe modulo $\sigma(E)$. Pour tout $s \in \mathbb{N}$ :

1) Si $p \mid i_{0}(\gamma)$, alors $v_{0}\left(\tau^{s} * \bar{x}\right)=v_{0}(\bar{x})+s i_{0}(\gamma)$;

2) Si $p$ ne divise pas $i_{0}(\gamma)$, si $i_{1}(\gamma)<(2 p-1) i_{0}(\gamma)$ et si $v_{0}(\bar{x}) \equiv i_{0}(\gamma)$ $(\bmod p)$, alors $v_{0}\left(\tau^{s} * \bar{x}\right)=v_{0}(\bar{x})+\theta(s)$, où $\theta: \mathbb{Z} \rightarrow \mathbb{Z}$ est l'application définie par : pour tout $(q, r) \in \mathbb{Z}^{2}$, avec $0 \leq r<p-1$,

$$
\theta(q(p-1)+r)=q i_{1}(\gamma)-(q-r) i_{0}(\gamma)
$$

Preuve. - On peut supposer que $\bar{x} \neq 0$, donc que $v_{0}(\bar{x})$ est un entier premier à $p$.

1) Soit $x$ un relèvement de $\bar{x}$ dans $E$ tel que $v(x)=v_{0}(\bar{x})$. Si $p$ divise $i_{0}(\gamma)$, on voit, par récurrence sur $s$, que, pour tout $s \in \mathbb{N}$,

$$
v\left(\tau^{s} * x\right)=v(x)+s i_{0}(\gamma)
$$

TOME $126-1998-\mathrm{N}^{\circ} 4$ 
comme c'est un entier premier à $p$, on a

$$
v_{0}\left(\overline{\tau^{s} * x}\right)=v_{0}\left(\tau^{s} * \bar{x}\right)=v_{0}(\bar{x})+s i_{0}(\gamma) .
$$

2) Supposons donc $i_{0}(\gamma)$ premier à $p$. En procédant par récurrence, on peut supposer que $s=q(p-1)+r \geq 1$ et que l'assertion est prouvée pour tous les entiers naturels $<s$. Distinguons deux cas :

a) Si $r \neq 0$, on a :

$$
v_{0}\left(\tau^{s-1} * \bar{x}\right)=v_{0}(\bar{x})+\theta(s-1)=v_{0}(\bar{x})+q i_{1}(\gamma)-(q-r+1) i_{0}(\gamma) .
$$

Comme $i_{1}(\gamma) \equiv i_{0}(\gamma)$ modulo $p$, on a

$$
v_{0}\left(\tau^{s-1} * \bar{x}\right) \equiv v_{0}(\bar{x})+(r-1) i_{0}(\gamma) \equiv r i_{0}(\gamma) \not \equiv 0 \quad(\bmod p)
$$

et on peut choisir un relèvement $z$ de $\tau^{s-1} * \bar{x}$ dans $E$ tel que $v(z)=$ $v_{0}\left(\tau^{s-1} * \bar{x}\right)$. D'après le lemme précédent, on a

$$
\begin{aligned}
v(\tau * z) & =v(z)+i_{0}(\gamma)=v_{0}(\bar{x})+q i_{1}(\gamma)-(q-r+1) i_{0}(\gamma)+i_{0}(\gamma) \\
& =v_{0}(\bar{x})+q i_{1}(\gamma)-(q-r) i_{0}(\gamma) \\
& =v_{0}(\bar{x})+\theta(s)
\end{aligned}
$$

Mais cet entier est congru modulo $p$ à $(r+1) i_{0}(\gamma)$ donc premier à $p$ et on a bien $v_{0}\left(\tau^{s} * \bar{x}\right)=v_{0}(\overline{\tau * z})=v(\tau * z)=v_{0}(\bar{x})+\theta(s)$.

b) Si $r=0$, alors $s \geq p-1$ et

$$
v_{0}\left(\tau^{s-(p-1)} * \bar{x}\right)=v_{0}(\bar{x})+(q-1)\left(i_{1}(\gamma)-i_{0}(\gamma)\right) \text {. }
$$

C'est un entier congru à $i_{0}(\gamma)$ modulo $p$ donc premier à $p$ et on peut choisir un relèvement $y$ de $\tau^{s-(p-1)} * \bar{x}$ dans $E$ tel que $v(y)=v_{0}\left(\tau^{s-(p-1)} * \bar{x}\right)$.

Le lemme précédent nous montre, par récurrence sur $n$, que pour tout entier $n$ vérifiant $0 \leq n \leq p-1, v\left(\tau^{n} * y\right)=v(y)+n i_{0}(\gamma)$. En particulier,

$$
v\left(\tau^{p-1} * y\right)=v(y)+(p-1) i_{0}(\gamma) \equiv 0 \quad(\bmod p) .
$$

On peut donc écrire $\tau^{p-1} * y=w_{0}+w$ avec $w_{0} \in \sigma(E)$ et $w \in E$ tels que :

$$
v\left(w_{0}\right)=v\left(\tau^{p-1} * y\right)
$$

et ou bien $w=0$ ou bien $v(w) \not \equiv 0(\bmod p)$ et $v(w)>v\left(w_{0}\right)$.

On voit que $w$ est un relèvement dans $E$ de $\tau^{s} * \bar{x}$ et que $v_{0}\left(\tau^{s} * \bar{x}\right)=v(w)$.

L'assertion 2) du lemme précédent montre que :

$$
v\left(\tau * w_{0}\right) \geq v\left(w_{0}\right)+p i_{0}(\gamma)=v(y)+(2 p-1) i_{0}(\gamma)>v(y)+i_{1}(\gamma)
$$

tandis que l'assertion 1) montre que $v\left(\tau^{p} * y\right)=v(y)+i_{1}(\gamma)$.

Comme $\tau^{p} * y=\tau * w_{0}+\tau * w$, on a $v(\tau * w)=v\left(\tau^{p} * y\right)$ ou encore

$$
v(w)+i_{0}(\gamma)=v(y)+i_{1}(\gamma)=v_{0}(\bar{x})+q i_{1}(\gamma)-(q-1) i_{0}(\gamma) .
$$

Donc $v_{0}\left(\tau^{s} * \bar{x}\right)=v(w)=v_{0}(\bar{x})+q i_{1}(\gamma)-q i_{0}(\gamma)=v_{0}(\bar{x})+\theta(s)$. 


\subsection{Fin de la preuve du théorème 5.1.}

On note $E_{*}$ le corps $E$ muni de sa structure de $k[[\tau]]$-module définie par l'action $*$.

Compte-tenu du lemme 5.5, la proposition 5.2 et son corollaire nous permettent, quitte à remplacer $K$ par l'extension de degré $p^{n}$ de $K$ contenue dans $L$, avec $n$ suffisamment grand et $\gamma$ par $\gamma^{p^{n}}$ de supposer que $i_{1}(\gamma)-i_{0}(\gamma)=p e_{K}$ et que $i_{1}(\gamma)<(2 p-1) i_{0}(\gamma)$

Soit $\mathfrak{m}$ l'idéal maximal de l'anneau des entiers de $E$. Pour tout $t \in \mathbb{Z}$, notons $\overline{\mathfrak{m}}^{t}$ l'image de l'idéal fractionnaire $\mathfrak{m}^{t}$ dans le quotient $E / \sigma(E)$.

On distingue alors deux cas :

a) Le cas où $p$ divise $i_{0}(\gamma)$. - On voit, par récurrence sur $s \in \mathbb{N}$, que $v\left(\tau^{s}(\pi)\right)=1+s i_{0}(\gamma)$, d'où l'on déduit que $i_{n}(\gamma)=p^{n} i_{0}(\gamma)$ pour tout entier naturel $n$. La suite des $i_{n}(\gamma) / p^{n}$ est donc constante et, d'après le corollaire $5.2, i_{0}(\gamma)=p e_{K} /(p-1)$.

On en déduit que l'ensemble $I$ des entiers $i$ premiers à $p$ tels que $0<i<i_{0}(\gamma)$ a $e_{K}$ éléments.

Pour $t \in \mathbb{Z}, i \in I$ et $s \in \mathbb{N}$, posons :

$$
\nu_{i, s}^{t}=p t+i+s i_{0}(\gamma)
$$

Pour $t$ fixé, on voit que les entiers $\nu_{i, s}^{t}$ sont deux à deux distincts et parcourent l'ensemble des entiers premiers à $p$ supérieurs à $p t$.

Comme, pour tout $i \in I$ et tout $t \in \mathbb{Z}, p t+i$ est premier à $p$, l'assertion 1) du lemme 5.8 montre que :

$\forall s \in \mathbb{N}, \forall i \in I, \forall t \in \mathbb{Z}, \quad v_{0}\left(\tau^{s} * \overline{\pi^{p t+i}}\right)=p t+i+s i_{0}(\gamma)=\nu_{i, s}^{t}$

On en déduit, en appliquant le lemme 5.4, que pour tout $t \in \mathbb{Z}$ :

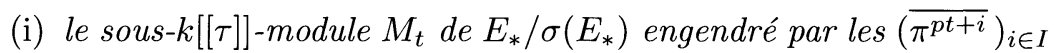
est libre de rang $e_{K}$;

(ii) $M_{t}=\overline{\mathfrak{m}}^{p t}$ et $\tau *\left(M_{t}\right)=M^{t+i_{0}(\gamma) / p}$.

Comme $E_{*} / \sigma\left(E_{*}\right)$ est la réunion des $M_{t}$, le théorème 5.1 s'en déduit facilement.

b) Le cas où $p$ ne divise pas $i_{0}(\gamma)$. - Posons cette fois-ci

$$
I=\left\{i \in \mathbb{Z} \mid i \equiv i_{0}(\gamma)(\bmod p) \text { et } i_{0}(\gamma) \leq i<i_{0}(\gamma)+e_{K} p\right\}
$$

C'est encore un ensemble à $e_{K}$ éléments.

TOME $126-1998-\mathrm{N}^{\circ} 4$ 
Pour $t \in \mathbb{Z}, i \in I$ et $s \in \mathbb{N}$, posons :

$$
\nu_{i, s}^{t}=p t+i+\theta(s)
$$

Si $s \in \mathbb{N}$ et si $s=q(p-1)+r$ est la division euclidienne de $s$ par $p-1$, on a :

$$
\theta(s)=q i_{1}(\gamma)-(q-r) i_{0}(\gamma)=q p e_{K}+r i_{0}(\gamma)
$$

On en déduit que, pour $t$ fixé, la famille des $\nu_{i, s}^{t}$ est en bijection avec celle des

$$
\mu_{i, q, r}^{t}=p t+i+q p e_{K}+r i_{0}(\gamma)
$$

pour $i \in I, q \in \mathbb{N}$ et $r \in \mathbb{N}$ vérifiant $0 \leq r<p-1$.

On vérifie qu'ici encore, pour $t$ fixé, ces entiers sont deux à deux distincts et sont tous premiers à $p$. L'assertion 2) du lemme 5.8 montre que

$$
\forall s \in \mathbb{N}, \forall i \in I, \forall t \in \mathbb{Z}, \quad v_{0}\left(\tau^{s} * \overline{\pi^{p t+i}}\right)=p t+i+\theta(s)=\nu_{i, s}^{t}
$$

et donc que

(i) le sous- $k[[\tau]]-$ module $M_{t}$ de $E_{*} / \sigma\left(E_{*}\right)$ engendré par les $\left.\overline{\left(\pi^{p t+i}\right.}\right)_{i \in I}$ est libre de rang $e_{K}$.

En regardant les valeurs prises par les entiers $\mu_{i, q, r}^{t}$, on vérifie aussi que

(ii) $M_{t}$ est contenu dans $\overline{\mathfrak{m}}^{p t+i_{0}(\gamma)}$ et contient $\overline{\mathfrak{m}}^{p t+p i_{0}(\gamma)}$. De plus, $\tau *\left(M_{t}\right)$ contient $\overline{\mathfrak{m}}^{p\left(t+e_{K}+i_{0}(\gamma)\right)}$.

Ici encore $E_{*} / \sigma\left(E_{*}\right)$ est la réunion des $M_{t}$ et le théorème 5.1 s'en déduit.

\section{BIBLIOGRAPHIE}

[Fo1] Fontaine (J.-M.). - Représentations p-adiques des corps locaux. The Grothendieck Festschrift, Birkhäuser, Boston, t. 2, 1991, p. 249309.

[Fo2] Fontaine (J.-M.). — Le corps des périodes p-adiques, exposé séminaire I.H.E.S. 1988, Périodes p-adiques, Astérisque 223, 1994 , p. 59-111. 
[Mil] Milne (J.S.). - Arithmetic duality theorems, Perspectives in Math., Academic Press, t. 1, 1986.

[Poi] Poitou (G.). - Cohomologie galoisienne des modules finis, Dunod.

[Sen] Sen (S.) On automorphisms of local fields. - Ann. of Math. (2), t. 90, 1969 , p. 33-46.

[Se1] Serre (J.-P.). - Corps locaux, $2^{\mathrm{e}}$ éd. - Hermann, 1968.

[Se2] Serre (J.-P.). - Cohomologie Galoisienne, $5^{\mathrm{e}}$ éd, Lecture Notes Math., Springer, t. 5, 1994.

[Tat] Tate (J.). - Duality theorems in Galois cohomology over number fields. - Proc. Int. Congress Math. Stokholm, 1962, p. 288-295.

[Wac] WACH (N.). - Représentations p-adiques potentiellement cristallines, Bull. Soc. Math. France, t. 124, 1996, p. 375-400.

[Win] Wintenberger (J.-P.). - Le corps des normes de certaines extensions infinies de corps locaux; applications, Ann. Sci. École Normale Supérieure (4), t. 16, 1983, p. 59-89.

[Wym] Wyman (B.F.). - Wildly ramified gamma extensions, Amer. J. Math., t. 91, 1969, p. 135-152. 